\title{
DEFORMATION MAPS FOR BOLTED T-STUBS
}

\author{
A.C. Faralli ${ }^{1}$, P.J. Tan ${ }^{2}$, G.J. McShane ${ }^{3}$ and P. Wrobel ${ }^{4}$
}

\section{ABSTRACT}

Deformation maps on the plastic and ultimate failure of T-stubs with a single bolt-row in tension are developed. The maps condense a large body of information within a two dimensional parameters space onto which different modes of deformation, including the régime boundaries, are plotted for any practical combination of geometric and material properties encountered in a T-stub. Its fidelity is demonstrated with experimental data from literature, and through a detailed parametric investigation by high-fidelity finite element analysis. The predictive capability of two existing analytical models are also assessed against predictions by the finite element model. Their shortcomings, and applicability, are critically assessed and discussed.

Keywords: T-stubs, Eurocode 3, Deformation maps, Failure modes, Joints.

\section{INTRODUCTION}

A bolted end plate connection is a class of moment resisting connections that is widely encountered in steel-framed structures. Its overall resistance is offered through a combination of tensile forces that act in the bolts adjacent to one flange,

\footnotetext{
${ }^{1}$ Research Assistant, Department of Mechanical Engineering, University College London, Torrington Place, London WC1E $7 \mathrm{JE}$.

${ }^{2}$ Associate Professor, Department of Mechanical Engineering, University College London, Torrington Place, London WC1E 7JE. Corresponding Author. Email: pj.tan@ucl.ac.uk

${ }^{3}$ Senior Lecturer, Department of Engineering, University of Cambridge, Trumpington Street, Cambridge CB2 1PZ.

${ }^{4}$ Emeritus Professor, Department of Mechanical Engineering, University College London, Torrington Place, London WC1E 7JE.
} 
and compressive forces experienced by the bearing at the other. Unless significant catenary actions develop in its adjoining beam, these tensile and compressive forces are typically assumed to be equal and opposite. In general, the rotational capacity of any bolted joint is limited by the deformation within its tension zone which comprises of the column flange, end-plate and bolts in tension. In Eurocode 3 (EN 1993-1-8 2005) - denoted hereinafter as EC3 - the tension zone is modelled by a T-stub and the ability to predict, a priori, its mode of deformation for a broad range of material (T-stub and bolt) and geometric combinations is integral to characterising the deformation capacity of a structural joint.

For T-stubs comprising of a single bolt-row in tension, three modes of failure, or régimes of deformation, can develop: mode 1 - complete yielding of the flange; mode 2 - bolt failure with yielding of the flange; and, mode 3 - bolt failure. Within the constitutive framework of limit analysis, Piluso et al. (2001) showed that each mode corresponds to a unique range of non-dimensional parameter $\beta$ (Eq. 1). To extend the aforesaid to include material strain-hardening and 'ultimate' failure prediction, Piluso et al. (2001) adopted a piece-wise linear approximation of the true stress-strain curve of the flange material. A disadvantage is that $\beta$ must be reevaluated - including the critical values corresponding to the transition between régimes $1 \rightarrow 2\left(\beta_{\mathrm{cr}}^{1 \rightarrow 2}\right)$ and $2 \rightarrow 3\left(\beta_{\mathrm{cr}}^{2 \rightarrow 3}\right)$ - each time a different combination of material and geometry (dimensions) is encountered. At present, there is no straightforward means to visualise, and represent, the different modes and their régime boundaries for a practical range of material and geometric parameters in a compact design space that would be useful to designers.

Failure (both plastic and ultimate) prediction using $\beta$ is a resistance-based approach that does not shed light on the deformation of the T-stub. To this end, 


\section{DEFORMATION MAPS}

The mode of deformation that develops in a T-stub is related to a non-dimensional parameter given by (Piluso et al. 2001)

$$
\beta=\frac{2 M}{m B}
$$

where $m$ is the distance from the axis of the bolt-hole to the plastic hinge at the 
flange-to-web connection; $M$ is the bending moment at the plastic hinges; and, $B$ is the tensile force in each bolt shown in Fig 1. Since $M=M\left(l_{e f f}, t_{p}, f_{y}\right)$ and $B=B\left(A_{s}, f_{u b}\right)$, the parametric dependence for $\beta$ can be expressed - by making use of dimensional analysis - as follows:

$$
\beta=f\left(\frac{l_{e f f}}{m}, \frac{f_{y}}{f_{u b}}, \frac{t_{p}^{2}}{A_{s}}\right)
$$

which can be re-arranged to give

$$
\frac{t_{p}^{2}}{A_{s}}=g\left(\beta, \frac{l_{e f f}}{m}, \frac{f_{y}}{f_{u b}}\right)
$$

where $A_{s}$ is the tensile stress area of the bolt; $f_{y}$ and $f_{u b}$ are the yield strength of the flange material and the ultimate strength of the bolt, respectively; and, $t_{p}$ is the flange thickness. The effective length $l_{e f f}$ is the notional width defined such that, at plastic collapse, the resistance of the flange - modelled as beams - is equivalent to a T-stub whose kinematic mechanism is determined by the yield line pattern that develops. Figure 2 shows the different possible yield line patterns according to EN 1993-1-8 (2005). A circular pattern arises due to localised action of the bolts; hence, they only develop in mode 1 where prying forces $Q_{1}$ are small compared to that $\left(Q_{2}\right)$ in mode 2. By contrast, non-circular patterns can develop in both modes 1 and 2, and are characterised by significant prying forces that can cause premature bolt failure. In EN 1993-1-8 (2005), prying forces are assumed to act along the edges of a T-stub at a distance $n$ from the bolt axis. According to McGuire and Winter (1978), a non-circular pattern will develop whenever the ratio $\lambda=n / m<1.25$. It is worth emphasising that both circular and non-circular patterns are three-dimensional (3D) yielding mechanisms since their hinge-line profile changes along the width of the T-stub, see Fig 2. By contrast, a beam yield-line pattern is not and it develops whenever the width $L$ is small, i.e. 
$L<4 m+1.25 n$. Here, only non-circular and beam patterns that induce significant prying forces are considered. Note that $l_{\text {eff }} / m$ in Eq. 3 depends on the yield-line pattern that develops in the flange. For a non-circular pattern, $l_{\text {eff }} / m=4+1.25 \lambda$ (EN 1993-1-8 2005). In a beam pattern, it is assumed, according to standard specifications for HE beams (UNI 5397:1978 1978), that $L=2.5 \cdot m$.

To construct the maps, consider the plastic and ultimate failure of a T-stub separately. For a fully-plastic limit state, the corresponding $\beta$ is given by Piluso et al. 2001)

$$
\beta=\beta_{R d}=\frac{2 M_{p l, R d}}{m B_{R d}},
$$

where $B_{R d}$ is the design tensile resistance of the bolt given by (EN 1993-1-8 2005)

$$
B_{R d}=0.9 A_{s} f_{u b}
$$

and $M_{p l, R d}$ is the design flexural resistance of the flange given by EN 1993-1-8 2005)

$$
M_{p l, R d}=0.25 l_{e f f} t_{p}^{2} f_{y} .
$$

Substituting for $B_{R d}$ and $M_{p l, R d}$ in Eq. 4, and re-arranging according to the dimensionless groups in Eq. 3, one obtains:

$$
\begin{aligned}
& \frac{t_{p}^{2}}{A_{s}}=\frac{1.8}{4+1.25 \lambda} \beta_{R d}\left(\frac{f_{y}}{f_{u b}}\right)^{-1} \quad \text { Non-circular pattern } \\
& \frac{t_{p}^{2}}{A_{s}}=0.72 \beta_{R d}\left(\frac{f_{y}}{f_{u b}}\right)^{-1} \quad \text { Beam pattern }
\end{aligned}
$$

Note that the relevant expression for $l_{e f f} / m$ corresponding to its respective yieldline pattern, given earlier, was substituted into Eq. 7. For an ultimate limit state, (Piluso et al. 2001)

$$
\beta=\beta_{u}=\frac{2 M_{f}}{m B_{u}}
$$


Here, $B_{u}$ is the ultimate tensile resistance of the bolt given by (Piluso et al. 2001)

$$
B_{u}=A_{s} f_{u b}
$$

and $M_{f}$ is the bending moment at material fracture of the flange. Adopting an idealised true stress-strain relation of the flange material in Fig. 3 - note that $\sigma_{y}\left(=f_{y}\right)$ and $\sigma_{u}\left(=f_{u}\right)$ is the yield and ultimate tensile strength, respectively the bending moment at fracture can be expressed as (Piluso et al. 2001)

$$
\begin{array}{r}
\frac{M_{f}}{M_{y}}=\kappa=\frac{1}{2}\left[3-\left(\frac{\varepsilon_{y}}{\varepsilon_{f}}\right)^{2}\right]+\frac{1}{2} \frac{E_{h}}{E}\left(\frac{\varepsilon_{f}-\varepsilon_{h}}{\varepsilon_{y}}\right)\left(1-\frac{\varepsilon_{h}}{\varepsilon_{f}}\right)\left(2+\frac{\varepsilon_{h}}{\varepsilon_{f}}\right) \\
-\frac{1}{2} \frac{E_{h}-E_{f}}{E} \frac{\varepsilon_{f}-\varepsilon_{u}}{\varepsilon_{y}}\left(1-\frac{\varepsilon_{u}}{\varepsilon_{f}}\right)\left(2+\frac{\varepsilon_{u}}{\varepsilon_{f}}\right),
\end{array}
$$

where the corresponding moment at yield is

$$
M_{y}=\frac{l_{e f f} t_{p}^{2}}{6} f_{y} .
$$

The constant $\kappa$ in Eq. 10 is a function of the flange material so that $\kappa=1$ at fully plastic condition. Following the same procedure as before, one obtains

$$
\begin{aligned}
& \frac{t_{p}^{2}}{A_{s}}=\frac{3}{4+1.25 \lambda} \beta_{u} \frac{1}{\kappa}\left(\frac{f_{y}}{f_{u b}}\right)^{-1} \quad \text { Non-circular pattern } \\
& \frac{t_{p}^{2}}{A_{s}}=1.2 \beta_{u} \frac{1}{\kappa}\left(\frac{f_{y}}{f_{u b}}\right)^{-1} \quad \text { Beam pattern }
\end{aligned}
$$

where the ratio $\lambda \triangleq n / m$. Régime boundaries are obtained by substituting $\beta_{R d}$ and $\beta_{u}$ in Eqs. 7 and 12 with the corresponding expression for $\beta$ in Table 1. The final expression for the régime boundaries has the following general form:

$$
\frac{t_{p}^{2}}{A_{s}}=h(\lambda) \frac{1}{\kappa}\left(\frac{f_{y}}{f_{u b}}\right)^{-1}
$$

where $h(\lambda)$ is tabulated in Table 2. Deformation maps for plastic and ultimate failure of a T-stub - generated by plotting the régime boundaries (Eq. 13) in a 
plot of $t_{p}^{2} / A_{s}$ versus $\kappa f_{y} / f_{u b}$ - are shown in Figs. 4 and 5 , respectively. Notice that each boundary is an isoline corresponding to a constant $\lambda$ value. Any pair of geometric $\left(t_{p}^{2} / A_{s}\right)$ and material $\left(\kappa f_{y} / f_{u b}\right)$ parameters now uniquely locates a point on the 2D map. From the map, one is able to determine the deformation mode for a given value of $\lambda$ (or by interpolation between any two $\lambda$ values plotted in Figs. 4 and 5 , if required). Alternatively, the map allows a designer to select the combination of geometric and material parameters for a T-stub to deform in a desired mode. Note that only a single boundary demarcates the transition from mode $2 \rightarrow 3$ for the beam pattern since it is independent of $\lambda$. The fidelity of the maps will be validated later against experimental data from existing literature and numerical predictions by FE model to be developed next. It is worth emphasising that the deformation maps are only as accurate as the calculations/assumptions undertaken in their generation.

\section{FINITE ELEMENT MODELLING}

Three-dimensional finite element (FE) models are developed for T-stubs subjected to quasi-static tension with ABAQUS/Standard V6.13. Predictions will be validated against experimental data from three separate independent sources: viz. WT1 (Girão Coelho 2004), T1 (Bursi and Jaspart 1997) and T15 (Ribeiro et al. 2015). The acronyms correspond to that used in their respective original source. The aforesaid were performed for identical flange (S355 steel) and bolt (Grade 8.8) materials, but each have a different $\lambda$ (WT1: 0.9, T1: 1.0 and T15: 0.7). All three failed in either mode 1 or 2 depending on the non-dimensional parameter $t_{p}^{2} / A_{s}$ which is different for each data-set. It is worth highlighting that both WT1 and T1 contained two bolt-rows. Since they both develop a beam yield line pattern, 
3D effects can be neglected and interactions between the two bolt-rows need not be considered (EN 1993-1-8 2005); consequently, they can be treated as equivalent T-stubs with a single bolt row.

\section{Constitutive model}

All the flange and bolts in the experiments were constructed from S355 steel and Grade 8.8 bolt (of Young's modulus $E=210 \mathrm{GPa}$ and Poisson's ratio $\nu=$ 0.33), respectively. Their subsequent plastic responses are modelled using the conventional $J_{2}$ plasticity flow theory to allow implementation of the progressive degradation of material stiffness. Figure 6 shows the nominal, and corresponding true stress-strain curves of the S355 flange material. Characteristic points on the nominal and true stress-strain curves are indicated as follows: yield $(y)$, necking $(n)$, rupture $(r$ - the last point on the softening branch just before the stress drops to 0$)$ and fracture $(f)$. An index $i$ denotes a generic data point on the stress-strain curve (nominal or true) connecting points $y, n, r$ and $f$. The $i$-th data point of the plastic response is extracted from the nominal stress-strain curve through (ABAQUS 2009)

$$
\sigma_{i}= \begin{cases}\sigma_{i}^{\mathrm{nom}}\left(1+\varepsilon_{i}^{\mathrm{nom}}\right), & y \leq i<n \\ \sigma_{n}^{\mathrm{nom}}\left(1+\varepsilon_{i}^{\mathrm{nom}}\right), & n \leq i \leq f\end{cases}
$$

and

$$
\varepsilon_{i}^{\mathrm{pl}}=\ln \left(1+\varepsilon_{i}^{\mathrm{nom}}\right)-\varepsilon_{y},
$$

where $\sigma_{n}^{\text {nom }}$ is the nominal stress at necking and $\varepsilon_{y}$ is the strain at yield. To account for the effects of post necking strain localisation, we follow Pavlović et al. (2013) 
by defining the nominal strain $\varepsilon_{i}^{\text {nom }}$ as

$$
\varepsilon_{i}^{\mathrm{nom}}= \begin{cases}\Delta l_{i} / l_{i}, & y \leq i<n \\ \varepsilon_{i-1}^{\mathrm{nom}}+\left(\Delta l_{i}-\Delta l_{i-1}\right) / l_{i}, & n \leq i \leq f\end{cases}
$$

where $l_{i}$ - given in Eq. 17 - represents the gauge length at the $i$-th data point and $\Delta l_{i}=\varepsilon_{i}^{\text {nom }} l_{0}$. For a cylindrical tensile coupon of diameter $d$, the initial gauge length $l_{0}(=50 \mathrm{~mm})$ starts decreasing as the material softens. At the point of rupture, the gauge length becomes $l_{\mathrm{loc}}=0.5 d$ (Panontin and Sheppard 1999): here, $l_{l o c}=6 \mathrm{~mm}$. Following Pavlović et al. (2013), the reduction of the gauge length is assumed to obey a power law through the localisation rate factor $\left(\alpha_{L}=0.5\right)$ given by

$$
l_{i}= \begin{cases}l_{0}, & y \leq i<n \\ l_{0}+\left(l_{\mathrm{loc}}-l_{0}\right)\left[\left(\Delta l_{i}-\Delta l_{n}\right) /\left(\Delta l_{r}-\Delta l_{n}\right)\right]^{\alpha_{L}} & n \leq i \leq f,\end{cases}
$$

where $\Delta l_{n}=\varepsilon_{n}^{\text {nom }} l_{0}$ and $\Delta l_{r}=\varepsilon_{r}^{\text {nom }} l_{0}$ are the elongations of the gauge length at necking and rupture, respectively. The effects of strain localisation were not taken into account for the bolt since experiments by Girão Coelho (2004) have shown that the area reduction following the onset of necking affects the entire length of its shaft. Instead, the true stress-plastic strain curve obtained by Girão Coelho (2004) - this is plotted in Fig. 6- is used for the bolt.

\section{Damage modelling}

The damage initiation criterion by ABAQUS $(2009)$ is used to predict the onset of damage over a wide range of stress states, given by

$$
\omega_{d}=\int \frac{d \bar{\varepsilon}^{\mathrm{pl}}}{\varepsilon_{0}^{\mathrm{pl}}(\theta)}=1
$$


where $\omega_{d}$ is a state variable that increases monotonically with $\bar{\varepsilon}^{\mathrm{pl}}$ (expressed as a function of stress triaxiality $\theta$ ). Here, the equivalent plastic strain at the onset of damage $\bar{\varepsilon}_{0}^{\mathrm{pl}}$ is defined as (Pavlović et al. 2013)

$$
\bar{\varepsilon}_{0}^{\mathrm{pl}}(\theta)=\varepsilon_{n}^{\mathrm{pl}} \cdot \exp [-1.5(\theta-1 / 3)]
$$

where $\varepsilon_{n}^{\mathrm{pl}}$ is the true plastic strain at necking. In the post necking regime $(n \leq$ $i \leq f)$, the damage process is controlled by the evolution of the damage variable $D_{i}$ which is expressed as a function of the equivalent plastic displacement $\bar{u}_{i}^{\mathrm{pl}}$. Following Pavlović et al. (2013), $D_{i}$ is given by

$$
D_{i}=\left\{\begin{array}{rc}
\left(1-\bar{\sigma}_{i} / \sigma_{i}\right) \cdot 1.5, & n \leq i \leq r \\
1, & i=f
\end{array}\right.
$$

where $\sigma_{i}$ is the true stress (Eq. 14) and $\bar{\sigma}_{i}=\sigma_{i}^{\text {nom }}\left(1+\varepsilon_{i}^{\text {nom }}\right)$ for $y \leq i \leq f$. Notice that no data points were considered between points $r$ (rupture) and $f$ (fracture) in Fig. 6 since rupture is defined as the last point on the softening branch of the nominal stress-strain curve, i.e. $D_{i}=1$ beyond point $r . \bar{u}_{i}^{\mathrm{pl}}$ is given by (Pavlović et al. 2013)

$$
\bar{u}_{i}^{\mathrm{pl}}=\bar{u}_{f}^{\mathrm{pl}}\left(\varepsilon_{i}^{\mathrm{pl}}-\varepsilon_{n}^{\mathrm{pl}}\right) /\left(\varepsilon_{f}^{\mathrm{pl}}-\varepsilon_{n}^{\mathrm{pl}}\right), n \leq i \leq f
$$

where the equivalent plastic displacement at fracture $\bar{u}_{f}^{\mathrm{pl}}$ (in Eq. 21) is

$$
\bar{u}_{f}^{\mathrm{pl}}=\lambda_{\mathrm{S}} \lambda_{\mathrm{E}} L_{\mathrm{E}}\left(\varepsilon_{f}^{\mathrm{pl}}-\varepsilon_{n}^{\mathrm{pl}}\right)
$$

and $L_{\mathrm{E}}=\sqrt[3]{V}$ ( $V$ is the volume of the element) is the characteristic length of the element (ABAQUS 2009, Sui et al. 2017). The factor $\lambda_{E}$ must be calibrated to the element type used in the FE model. The dependence of $\bar{u}_{f}^{\mathrm{pl}}$ on the mesh size is removed by introducing an element size factor $\lambda_{S}$, which is obtained by reproducing the tensile test in FE with different element size of $L_{E, a}$, where $a \in[0, t]$ and $t$ is 
the number of element sizes considered. The reference element size is $L_{E, a}=L_{E, 0}$ for $a=0$. The final expression for $\lambda_{S}$ is (Pavlović et al. 2013)

$$
\lambda_{S}=\sqrt[3]{\frac{L_{E, 0}}{L_{E, a}}}, a \in[1, t] .
$$

It is worth emphasising that $\lambda_{E}$ is used to model damage evolution in the S355 steel. For the Grade 8.8 bolt, a linear damage evolution law is used for simplicity since it does not require the introduction of $\lambda_{E}$.

\section{Calibration of $\lambda_{E}$}

Tension loading of dog-bone specimens for the S355 steel were simulated to calibrate $\lambda_{E}$. Four specimens with cylindrical cross-sectional area, and dimensions stipulated by the ASTM Standard E8/E8M-15a (2015), are discretised with C3D8R (8 nodes linear continuum elements with reduced integration) elements of a uniform size throughout its gauge section. The element sizes considered were: $L_{E, 0}=0.89 \mathrm{~mm}$ (186 elements across diameter), $L_{E, 1}=1.00 \mathrm{~mm}$ (140 elements), $L_{E, 2}=1.25 \mathrm{~mm}$ (96 elements) and $L_{E, 3}=1.59 \mathrm{~mm}$ (60 elements). Each element size was chosen so that their aspect ratio is $\approx 1$. A displacement boundary condition of $0.05 \mathrm{~mm} / \mathrm{s}$ (corresponding to a nominal strain rate of $0.001 \mathrm{~s}^{-1}$ ) is applied to one end of the specimen, with the other end fully clamped, to simulate tensile loading. The true stress-strain curve in Fig. 6 is used and damage initiation follows Eq. 19 .

A flow chart summarising the procedure to calibrate $\lambda_{E}$ is shown in Fig. 7. An initial FE model discretised uniformly with C3D8R elements of size $L_{E, 0}$ is first considered with a trial $\lambda_{E}$ value. $\lambda_{S}$ is, then, evaluated - note that $\lambda_{S}=1$ (Eq. 23) for $L_{E, a}=L_{E, 0}-$ and Eqs. 20, 21 and 22 are used to obtain $D_{i}, \bar{u}_{i}^{\mathrm{pl}}$ and $\bar{u}_{f}^{\mathrm{pl}}$, respectively. The predicted stress-strain curve is then compared to its 
experimental counterpart. If their differences $\operatorname{Er}\left(\varepsilon_{r}^{\text {nom }}\right) \leq 2 \%$, then the trial $\lambda_{E}$ value is satisfactory and the same procedure repeated for $L_{E, 1}, L_{E, 2}$ and $L_{E, 3}$. If, however, $\operatorname{Er}\left(\varepsilon_{r}^{\text {nom }}\right)>2 \%$ for any $L_{E, a}$, the initial value of $\lambda_{E}$ is updated and the simulations repeated for all $L_{E, a}$. Pavlović et al. (2013) have shown that $\lambda_{E} \in[2.5,3.2]$ for C3D8R elements and the final value to use depends on the ductility of the material. Here, the calibrated value of $\lambda_{E}=2.5$ lies within the range stipulated by Pavlović et al. (2013).

The difference in the predicted nominal strain at rupture $\varepsilon_{r}^{\text {nom }}$ with experiment is less than $2 \%$ for all $L_{E, a}$. A linear damage evolution law is assumed for the bolt, with an equivalent plastic displacement at fracture given by $\bar{u}_{f}^{\mathrm{pl}}=\varepsilon_{f} L_{\mathrm{E}}(\mathrm{ABAQUS}$ 2009). $L_{\mathrm{E}}=2 \mathrm{~mm}$ is used here so that there is a minimum of 12 to 16 nodes across the bolt diameter (Virdi 1999); and, $\varepsilon_{f}=0.13$ follows from Girão Coelho (2004). Figure 8 shows the damage initiation and evolution curves that were implemented into the FE models.

\section{Simulations of T-stub in tension and validation}

Figure 9 shows a schematic of the tensile test simulated by FE. Recall that WT1 and T1 comprises of two bolt-rows; hence, only a quarter of the T-stub was simulated and $u_{z}=0$ must be specified for the $x-y$ plane (Fig. 9a), unlike in T15. The bottom web is fully clamped and a displacement boundary condition of $\dot{u}_{y}=0.01 \mathrm{~mm} / \mathrm{s}$ (Girão Coelho 2004) was applied to the top web. Table 3 lists the value of each geometric parameter shown in Figure 9b. Since T15 was obtained from an IPE300 beam profile (Bursi and Jaspart 1997), there is no weld to be modelled. By contrast, both WT1 (Girão Coelho 2004) and T1 (Ribeiro et al. 2015) were constructed by welding two plates together. In our FE model, 
¡both welds and flange are assumed to be made of the same material Girão Coelho 2004, Girão Coelho et al. 2004, Ribeiro et al. 2013, Latour and Rizzano 2012). The same is also assumed for T15 given that no information was provided by Ribeiro et al. (2015) regarding the weld material. The entire FE model is discretised using C3D8R elements (Latour and Rizzano 2012; Latour et al. 2014). The bolts are modelled as a solid cylinder with an equivelent cross-sectional area of $A_{s}$ Bursi and Jaspart 1997). Figure 10a shows the results of three element sizes (4, 2 and $1.5 \mathrm{~mm}$ ) - the flange is meshed as in B4C5D4 of Fig. 10d (this will be justified later) - that were used to mesh the bolt. Since a $1.5 \mathrm{~mm}$ element predicted a similar ultimate displacement $\Delta_{u}$ - black circular dot in Fig. 10 - to its $2 \mathrm{~mm}$ counterpart, the latter was used to reduce computational cost. The mesh for the bolt, used in subsequent parametric study, is shown in Fig. 9d. To determine the mesh size for the flange, we partition the flange into four zones (A, B, C and D) as shown in Fig. 10d. Zone $\mathrm{A}$ is discretised using C3D8R elements of different sizes, while the rest of the flange was meshed as shown in B4C5D4 (Fig. 10d), and their corresponding results are shown in Fig. 10p. After this, three mesh densities were tested for zones B, C and D - see Fig. 10 d - where the number of elements along the $\mathrm{x}$-axis is given after each letter. Figure 10 a shows a negligible difference between the predicted $\Delta_{u}$ by B4C5D4 and B6C10D4. Hence, Region B is discretised with four elements across the circle; while region $\mathrm{C}$ with four elements between regions A \& B and between regions B \& D. Region D is discretised with 4 elements. The mesh for the flange, used in subsequent parametric study, is shown in Fig. 9c. Surface-to-surface contact formulation with small sliding is prescribed for all contact pairs - top and bottom flange, flanges and head/nut of the bolt, bolt shank and hole - with a coefficient of Coulomb friction $\mu=0.25$ (Bursi and 
Jaspart 1997).

Figure 11 compares the predicted deformation history of the T-stubs to its experimental counterpart. The resistance $\left(F_{u}\right)$ of a T-stub is defined as the maximum resultant reaction force acting at the bottom web and its corresponding displacement is the ultimate displacement $\left(\Delta_{u}\right)$ - they are shown in Fig. 11 by red (experiment) and black (FEM) dots. The predicted force-displacement $(F-\Delta)$ curve closely matches that of the experiments where the percentage differences for $F_{u}$ (WT1: $3.4 \%$, T1: 3.1\%, T15: 5.3\%) and $\Delta_{u}$ (WT1: $0.7 \%, \mathrm{~T} 1: 5.1 \%, \mathrm{~T} 15:$ $2.6 \%)$ are small. In addition, comparison can also be made for the bolt elongation $\Delta_{b}$ in WT1 (note that this was not measured in T1 and T15) as shown in Fig. 11d. The discrepancy in the final bolt elongation between experiment and FE arises because the former was halted at $\Delta_{b}=0.4 \mathrm{~mm}$ to prevent equipment damage (Girão Coelho 2004). Notwithstanding, a good general agreement - differences of less than $5.1 \%$ - is observed until $\Delta_{b}=0.4 \mathrm{~mm}$. The mode of failure is also successfully predicted by the FE models; this is evident from the distributions of equivalent plastic strain $\left(\varepsilon^{\mathrm{pl}}\right)$ and damage variable $\left(D_{i}\right)$ shown in Fig. 11 . Note that the contour plots for the flange and bolt correspond to the ultimate displacement. Both WT1 and T15 failed in mode 2. A plastic hinge also develops at the weld-toe in the FE model which is highlighted in Figs. 11 a and c: here, $\varepsilon^{\mathrm{pl}}$ exceeds the threshold of 0.05 through the thickness, as suggested by Ribeiro et al. (2015). In Figs. 11a and c, the damage variable $D_{i}=1$ is reached in the bolt; consequently, elements are removed from the mesh. Specimen T1 developed two plastic hinges; at both the weld-toe and bolt hole. However, Fig. 11b also shows that $D_{i}$ is close to unity in the bolt. This suggests that the bolt had undergone significant plastic deformation which agrees with observations by Bursi and Jaspart (1997) who suggested that 
the actual failure mechanism is between mode 1 and 2 . The location of fracture in the experiment was not indicated by Bursi and Jaspart (1997); however, the value of $D_{i}$ in our FE model suggests that bolt fracture is imminent.

\section{RESULTS}

The FE model is now employed in a parametric study to investigate how the deformation capacity of a T-stub is affected by its geometric and material parameters; and, the results will also be used to critically assess the accuracy, and limitations, of two existing analytical models developed by Piluso et al. (2001) and Francavilla et al. (2016).

\section{Parametric study}

\section{Geometry and material properties}

Figure $9 \mathrm{~b}$ shows a schematic of the T-stub and the geometric dimensions considered. Note that the tensile response of a T-stub that develops beam yield line pattern had been extensively studied, both experimentally and numerically, by pthers; see, for example, Piluso et al. (2001), Girão Coelho (2004) and Ribeiro et al. (2015). In addition, analytical models also exist that could accurately predict their $F-\Delta$ relationship (Piluso et al. 2001; Francavilla et al. 2016). By contrast, there are relatively fewer studies on non-circular yield line patterns. For this reason, all the T-stubs here were sized to develop this; hence, their width $L$ must satisfy $L>4 m+1.25 n$. Since $B$ is fixed at $200 \mathrm{~mm}$ in all the models, $m$ and $n$ are varied to obtain three different $\lambda(\triangleq n / m)$ values as follows: (1) A maximum value of $\lambda_{\max }=1.25$ determined by considering the minimum standard bolt spacing of $p_{\text {min }}=98 \mathrm{~mm}$ for a HEA200 beam (UNI 5397:1978 1978). This is taken as a reference beam profile since it is characterised by a width which is identical to $B$ 
(200mm). Note that if $\lambda>\lambda_{\max }(=1.25)$, non-circular patterns will not develop according to McGuire and Winter (1978); (2) A minimum value of $\lambda_{\min }=0.9$ determined by considering the maximum allowable bolt spacing of $p_{\max }=14 t_{p}$ as stipulated in EN 1993-1-8 (2005); (3) An intermediate value of $\lambda_{\text {inter }}=1.1$ - this was selected to lie between $\lambda_{\max }$ and $\lambda_{\min }$. Three flange thicknesses $t_{p}(8,9$ and $10 \mathrm{~mm})$ and four bolt diameters $d_{b}(10,12,20$ and $27 \mathrm{~mm})$ are considered. Table 4 tabulates the combinations of dimensions that were modelled.

Four different grades of structural steel (S235, S275, S355 and S450) are modelled for the flange material. Their respective true stress-strain curve is represented using a piece-wise approximation similar to Piluso et al. (2001) in Fig. 3. Key values of stresses $\left(\sigma_{y}\right.$ and $\left.\sigma_{u}\right)$, strains $\left(\varepsilon_{y}, \varepsilon_{h}, \varepsilon_{u}\right.$ and $\left.\varepsilon_{f}\right)$ and moduli $\left(E, E_{h}\right.$ and $\left.E_{f}\right)$ are tabulated in Table 5. For the S450 steel, $\sigma_{y}$ and $\sigma_{u}$ were obtained from EN 1993-1-8 (2005), $E_{f}=\sigma_{u}$ (Piluso et al. 2001) and $\varepsilon_{f}=0.17$ (EN 10025-2 2004); and, $E, E_{h}, \varepsilon_{y}, \varepsilon_{h}$ and $\varepsilon_{u}$ are assumed to be identical to those of S355. The damage initiation criterion for each steel is given by Eq. 19 and their corresponding values tabulated in Table 6. Damage evolution is modelled as shown in Fig. 8 for all the four grades of steel because the actual nominal stress-strain curves were not provided by Girão Coelho (2004) for S235, S275 and S450. This is an acceptable assumption given that all four steels are characterised by similar $\varepsilon_{u}$ and $\varepsilon_{f}$ (Table 5). The same Grade 8.8 bolt is used throughout and is modelled as previously described.

\section{Results and Discussions}

Results of the parametric study are plotted in Fig. 12. Analytical predictions by Piluso et al. (2001) and Francavilla et al. (2016) are then compared to the 
FE predictions of the ultimate displacement $\Delta_{u}$ and failure mode for $\lambda_{\max }=1.25$ in Table 4. Since the analytical models are, hitherto, mostly applied only to $\lambda \approx 1$, comparison will be made here for $\lambda_{\max }=1.25$. The deformation capacity of a T-stub is charactersied by its non-dimensional ultimate displacement $\delta \triangleq$ $\Delta_{u} / t_{p}$, and this is plotted against $\Gamma \triangleq \kappa f_{y} / f_{u b} \cdot t_{p}^{2} / A_{s}$. Notice that $\Gamma$ is a product of two dimensionless groups that were previously used to delineate the régime boundaries. From Eq. 13 , it is clear that $\Gamma=h(\lambda)$; hence, for a constant $\lambda$, the régime boundaries depend only on $\Gamma$.

Figure 12 shows a general reduction in the deformation capacity $\delta$ with $\Gamma$ and/or when the mode switches from 1 to 3 . It is hardly surprising that the ductility of a T-stub in mode 1 is highest due to the collapse mechanism it develops. And, since the collapse mechanism is affected by both geometric and material parameters (EN 1993-1-8 2005), the ductility of T-stubs is highly dependent on the dimensionless parameters $t_{p}^{2} / A_{s}$ and $k f_{y} / f_{u b}$. Notice that the data points for mode 1 are much more disperse, further confirming the sensitivity of ductility to geometric parameters and material properties. By contrast, mode 3 deformation is dictated by the deformation of the bolts and is, consequently, less sensitive to geometric and material properties of the flange.

Table 4 tabulates the value of $\delta$ for each T-stub. It can be seen that, for a constant $\kappa f_{y} / f_{u b}, \delta$ increases for T-stubs constructed with a weak flange and strong bolts. These T-stubs fail predominantly in either mode 1 or 2 . By contrast, T-stubs with strong flanges and weak bolts deform primarily in mode 3 , and they have low deformation capacity $(\delta)$ that is nearly constant for $\Gamma>1.5$. Furthermore, Table 4 also shows that, for the same combination of $t_{p}$ and $A_{s}, \delta$ reduces with increasing $\kappa f_{y} / f_{u b}$ for all modes of failure. In addition, the effects of $\kappa f_{y} / f_{u b}$ on $\delta$ is 
greatest for $\lambda=\lambda_{\min }$ (mode 1: $5.3 \leq \delta \leq 6$ and mode 2: $1.9 \leq \delta \leq 4.5$ ) compared to $\lambda_{\text {inter }}$ (mode 1: $4.9 \leq \delta \leq 5.5$ and mode $2: 1.9 \leq \delta \leq 4.0$ ) and $\lambda_{\max }$ (mode 1: $4.6 \leq \delta \leq 5.1$ and mode $2: 1.4 \leq \delta \leq 3.6)$. This is because when $\lambda=\lambda_{\min }$, $n$ is small compared to $m$ which implies that the bolt spacing $p$ is large. Girão Coelho (2004) observed that a large $p$ is responsible for a reduction of the T-stub stiffness because the flange is not as rigidly constrained between the weld-toe and bolt line compared to cases where $\lambda=\lambda_{\max }(n>>m)$. Increasing stiffness of a T-stub is accompanied by a consequential increase in its ductility - T-stubs with $\lambda=\lambda_{\min }$ are more compliant - which leads to comparatively higher ductility of the T-stub (Girão Coelho 2004). By contrast, the effects of both $t_{p}^{2} / A_{s}$ and $\kappa f_{y} / f_{u b}$ are negligible on $\delta$ for large $\Gamma$ as the data points eventually flatten out.

Table 4 also compares the FE results for $\lambda=\lambda_{\max }$ to analytical predictions by Piluso et al. (2001) and Francavilla et al. (2016). In general, both models under-predict $\delta$ in mode 1 because the deformation mechanism was assumed to be two-dimensional. This simplification is only valid for a beam yield line pattern; instead, a non-circular yield line pattern characterised by 3D effects develops in the flange. The model by Piluso et al. (2001) over-predicts the displacement in mode 2 because it neglects the compatibility condition between the elongation of the bolt and the deformation of the flange; this was subsequently addressed by Francavilla et al. (2016). Notwithstanding, discrepancies remain between FE and analytical predictions in mode 2 because geometric non-linearities were neglected in both analytical models. However, the difference between FE and analytical predictions is small in mode 3 , which suggests that both analytical models are, in general, accurate if applied to T-stubs that undergo small displacements. The good agreement in mode 3 is also, partly, because of the insensitivity of mode 3 
deformation to the yield line pattern that develops.

\section{Failure deformation maps - validation}

The fidelity of the deformation maps is demonstrated for non-circular yield line pattern in Fig. 13 by plotting the data from the parametric study; and for the beam yield line pattern with experimental data from Girão Coelho (2004), Piluso et al. (2001), Bursi and Jaspart (1997) and Ribeiro et al. (2015). Plastic (dotted lines) and ultimate (solid lines) régime boundaries are plotted for $\lambda_{\min }$ (Fig. 13 a), $\lambda_{\text {inter }}$ (Fig. 13b) and $\lambda_{\max }$ (Fig. 13c). Note that the boundaries corresponding to mode $2 \rightarrow 3$ for $\lambda_{\max }$ lie below the ones for $\lambda_{\min }$, similarly in Figs. 4 and 5 . This is because T-stubs with a smaller $\lambda$ value tend to be more ductile; consequently, they are more likely to fail either in mode 1 or 2 . Only a limited combinations of $t_{p}^{2} / A_{s}$ and $f_{y} / f_{u b}$ causes mode 3 failure. The plastic failure mode predicted by the map is, in general, conservative since T-stubs that fail in mode 1 (or 2) were predicted to fail in mode 2 (or 3). This is unsurprising given that the régime boundaries in the plastic failure map were constructed within the constitutive framework of limit analysis (for the flange material). By contrast, the ultimate régime boundaries were constructed by assuming a linear piece-wise approximation of the stress-strain curve (Fig. 3) which gives a better prediction of the failure mode. The data points indicated by blue arrows in Fig. 13 - they were identified by their row number in Table 4 and the flange material - are outliers due to the approximate nature of the piecewise idealisation of the flange material. Apart from the outliers, the failure maps predict well the mode of deformation predicted by the parametric study.

The maps also demonstrate how failure mode is influenced by $t_{p}^{2} / A_{s}$ and $\kappa f_{y} / f_{u b}$. Increasing $t_{p}^{2} / A_{s}$ leads to a shift in the mode of failure from 1 to 3 which is evident 
from the columns of data - each column corresponds to a constant $\kappa f_{y} / f_{u b}-$ in Fig. 13. Similarly, increasing $\kappa f_{y} / f_{u b}$ at a constant $t_{p}^{2} / A_{s}$ value also leads to a shift towards a less ductile mode $(1 \rightarrow 2$ or $2 \rightarrow 3)$. It is worth noting that since $f_{u b}$ is identical in the parametric study, increasing $\kappa f_{y} / f_{u b}$ corresponds to a stronger flange. If the flange has an increased resistance to deformation, then the bolt is more likely to fail even for lower $t_{p}$ and higher $A_{s}$ values - see, for example, data points 19 (S450), 23 (S355) and 23 (S450) in Fig. 13.

To examine the effects of varying $A_{s}$ whilst keeping $t_{p}$ constant, consider the T-stub configurations 1 to 4 listed in Table 4. Figure 13(c) shows that a T-stub with a greater $A_{s}$ tends to fail in mode 1 - as exemplified by rows of data points labelled 3 and 4 - while a smaller $A_{s}$ leads to failure in mode 2 or 3 (exemplified by rows of data points 1 and 2). The plastic failure map - their régime boundaries are plotted as dotted lines - suggests that one would need to increase the diameter of the bolt in order for the T-stub to deform in mode 1 . This is in contrast to what the ultimate failure map would suggest. Hence, using the ultimate map prevents the over-sizing of bolts which is a common, yet expensive, strategy adopted by structural steel designers. If instead one focuses on configurations 1,5 and 9 - they are characterised by a constant $A_{s}$ - it is evident from Fig. 13(c) that increasing $t_{p}$ leads to a less ductile failure mode; see, for example, the rows of data points labelled 5 and 9 .

Table 7 compares the predicted deformation using the maps to existing experimental test data. It is clear that the plastic régime boundaries are excessively conservative as they tend to predict a mode 2 deformation for T-stubs failing in mode 1 . The ultimate boundaries are evidently more accurate and are capable of subdividing the geometric $\left(t_{p}^{2} / A_{s}\right)$ and material $\left(\kappa f_{y} / f_{u b}\right)$ parameters space into 
correct modes of failure that are consistent with the experimental data.

Finally, the maps are only as good as the theory (specifically, the constitutive idealisation of the flange material) used to construct them. But they are useful in spite of their inexactness for both designing and interpreting experiments, and in selecting and understanding the behaviour of T-stubs for engineering applications. And, by identifying the places where data or theory are poor, they can be systematically improved.

\section{CONCLUSIONS}

Failure deformation maps were constructed for the plastic and ultimate failure of a T-stub with a single bolt-row in tension. The maps allow to avoid iterative pre-design calculations by condensing a large body of information within the $2 \mathrm{D}$ parameters space $t_{p}^{2} / A_{s}-\kappa f_{y} / f_{u b}$. It was found that the failure mode is sensitive to the non-dimensional parameters $t_{p}^{2} / A_{s}$ and $\kappa f_{y} / f_{u b}$. The maps show that a ductile failure mode (mode 1 ) is induced by either decreasing $t_{p}$ or increasing $A_{s}$. The fidelity of the maps is demonstrated through existing experimental data and through a FE parametric investigation. It was shown that the analytical models by Piluso et al. (2001) and Francavilla et al. (2016) under-predict the ultimate displacement in mode 1 arising from the assumption of a beam yield line pattern. Both models are also shown to be accurate if applied to T-stubs undergoing small displacements were geometric non-linearities are negligible.

\section{ACKNOWLEDGMENTS}

The invaluable discussions with Dr Massimo Latour from the University of Salerno (Italy), Dr Costança Rigueiro from the Polytechnic Institute of Castelo Branco (Portugal), and Dr Joao Ribeiro of the University of Coimbra (Portugal) 
are gratefully acknowledged. The authors are also grateful to the Ministry of Defence, UK (David Manley - DE\&S Sea Systems Group) and QinetiQ (Robert Ball - Structures \& Survivability, Platform Design and Life Support IDT) for partial financial support.

\section{REFERENCES}

ABAQUS/Standard User's Manual, Version 6.9 (2009). Simulia. ASTM Standard E8/E8M-15a (2015). Standard Test Methods for Tension Testing of Metallic Materials. ASTM International, ASTM International, West Conshohocken, PA.

Bursi, O. and Jaspart, J. (1997). "Benchmarks for finite element modelling of bolted steel connections." J. Constr. Steel Res., 43(1), 17-42.

EN 10025-2 (2004). Hot rolled products of structural steels. Technical delivery conditions for non-alloy structural steels.

EN 1993-1-8 (2005). Eurocode 3: Design of steel structures - Part 1-8: Design of joints. CEN, Brussel: European Commitee for Standardization.

Francavilla, A., Latour, M., Piluso, V., and Rizzano, G. (2016). "Bolted T-stubs: A refined model for flange and bolt fracture modes." Steel Compos. Struct., 20, $267-293$.

Girão Coelho, A. (2004). "Characterization of the ductility of bolted end plate beam-to-column steel connections." Ph.D. thesis, Universidade de Coimbra, Coimbra, Portugal.

Girão Coelho, A., Bijlaard, F., Gresnigt, N., and Simões da Silva, L. (2004). "Experimental assessment of the behaviour of bolted T-stub connections made up of welded plates." J. Constr. Steel Res., 60(2), 269-311. 
Latour, M. and Rizzano, G. (2012). "Experimental Behavior and Mechanical Modeling of Dissipative T-Stub Connections." J. Struct. Eng., 138(2), 170-182.

Latour, M., Rizzano, G., Santiago, A., and Simões da Silva, L. (2014). "Experimental analysis and mechanical modeling of T-stubs with four bolts per row." J. Constr. Steel Res., 101, 158-174.

McGuire, W. and Winter, G. (1978). Steel structures. Prentice-Hall International series in Theoretical and Applied Mechanics (Eds.: NM Newmark and WJ Hall), Englewood Cliffs, N.J., USA.

Panontin, T. and Sheppard, S. (1999). Fatigue and fracture mechanics. ASTM.

Pavlović, M., Marković, Z., Veljković, M., and Budevac, D. (2013). "Bolted shear connectors vs. headed studs behaviour in push-out tests." J. Constr. Steel Res., 88, 134-149.

Piluso, V., Faella, C., and Rizzano, G. (2001). "Ultimate behavior of bolted Tstubs. I: Theoretical model." J. Struct. Eng., 127, 686-693.

Ribeiro, J., Rigueiro, C., and Santiago, A. (2013). "NUMERICAL ASSESSMENT OF T-STUB COMPONENT SUBJECT TO IMPACT LOADING." Congr. Métodos Numéricos en Ing., Bilbao, Spain.

Ribeiro, J., Santiago, A., Rigueiro, C., and Simões da Silva, L. (2015). "Analytical model for the response of T-stub joint component under impact loading." $J$. Constr. Steel Res., 106, 23-34.

Sui, W., Wang, Z., An, K., and Peter, O. (2017). "Damage analysis of doubler plate reinforced t-joints under uniaxial tensile load based on stress triaxiality." Tubular Structures XVI: Proceedings of the 16th International Symposium for Tubular Structures (ISTS 2017, 4-6 December 2017, Melbourne, Australia), CRC Press, 263. 
UNI 5397:1978 (1978). Prodotti finiti di acciaio laminati a caldo. Travi HE ad ali larghe parallele. Dimensioni e tolleranze. CEN.

Virdi, K. (1999). "Guidance on good practice in simulation of semirigid connections by the finite element method. In: Numerical simulation of semi-rigid connections by the finite element method." Report no., COST C1, Report of working group 6 - Numerical simulation, Brussels; 1-12, 


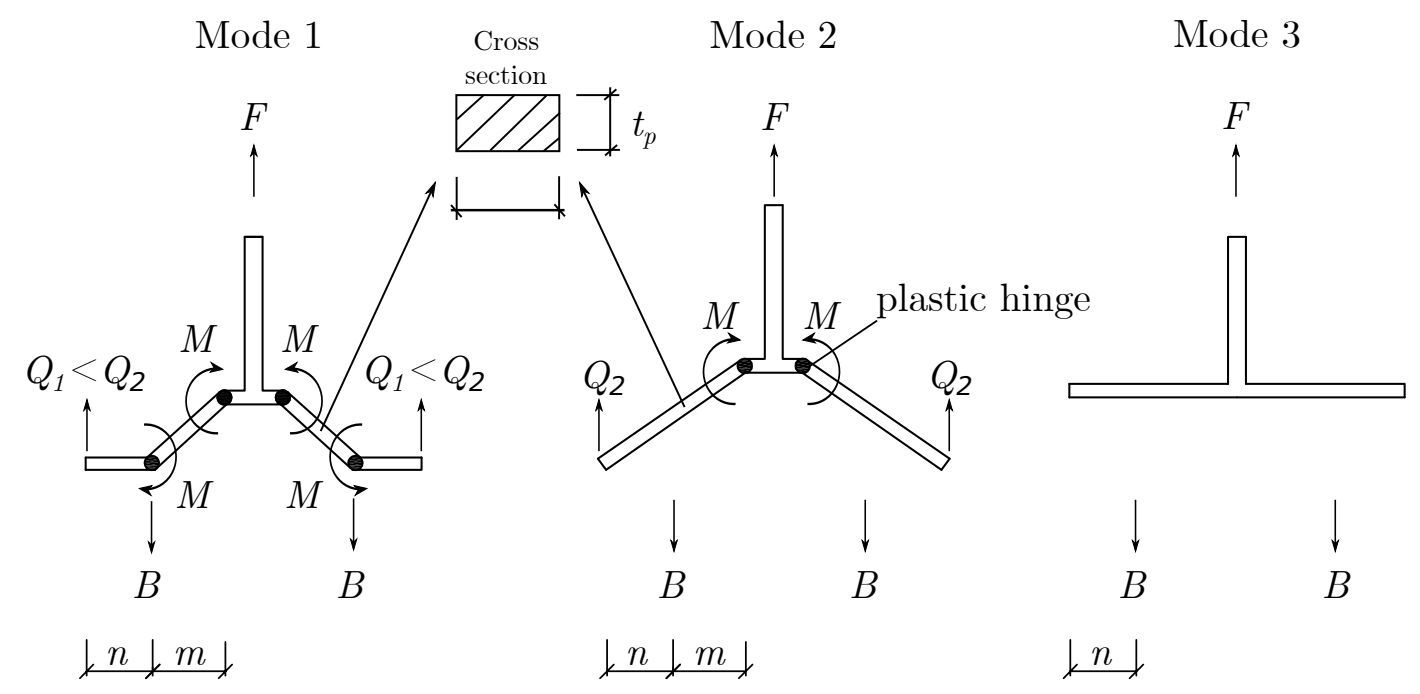

FIG. 1. Schematic of failure modes adapted from Ribeiro et al. (2015). $Q$ is prying force and $B$ is tensile force in the bolt. 
Modes 1 and 2

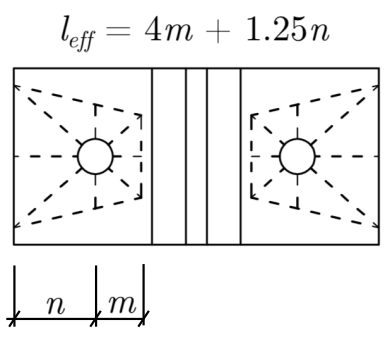

(a)
Mode 1

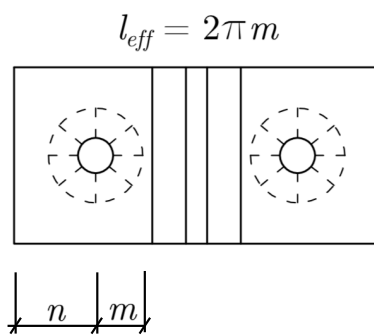

(b)
Modes 1 and 2

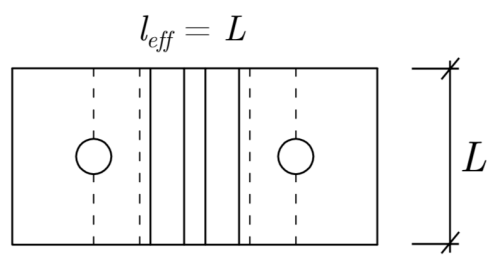

(c)

FIG. 2. Possible yield line patterns in a T-stub: (a) non-circular; (b) circular; and (c) beam. - - - shows hinge line and $L$ is the width (adapted from Girão Coelho (2004)). 


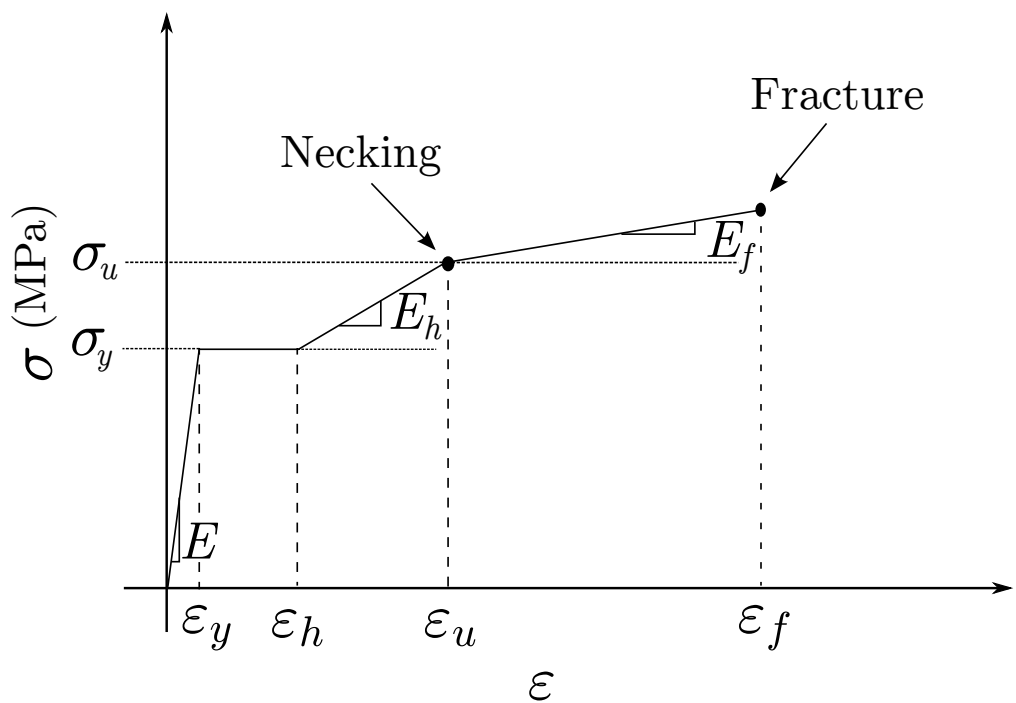

FIG. 3. Idealised piece-wise approximation of the true stress-strain curve of the flange material (Piluso et al. 2001). 


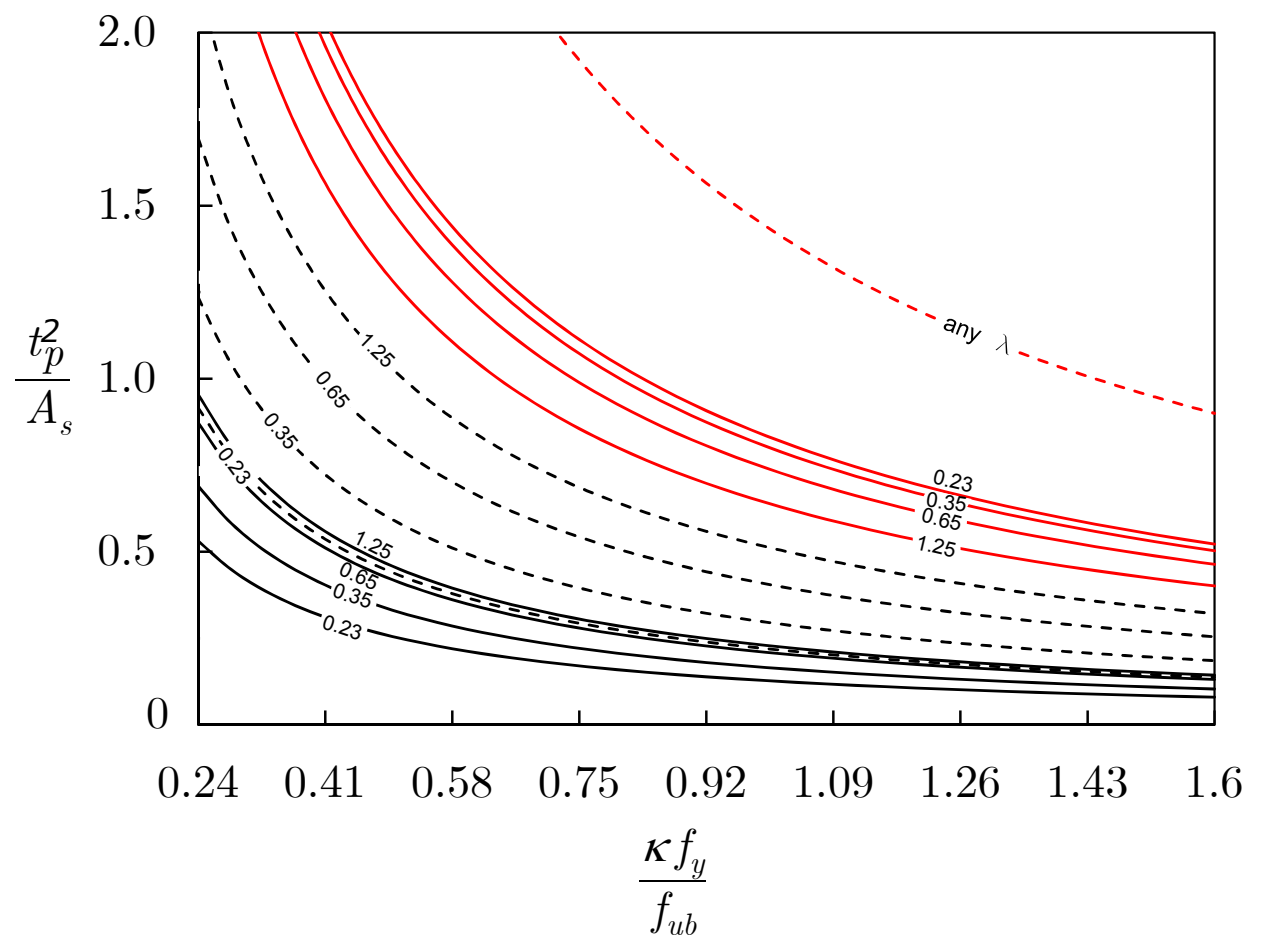

FIG. 4. Plastic failure map. Black and red isolines are régime boundaries at mode $1 \rightarrow 2$ and mode $2 \rightarrow 3$ transition, respectively. Solid lines for non-circular and dotted lines for beam yield line patterns. 


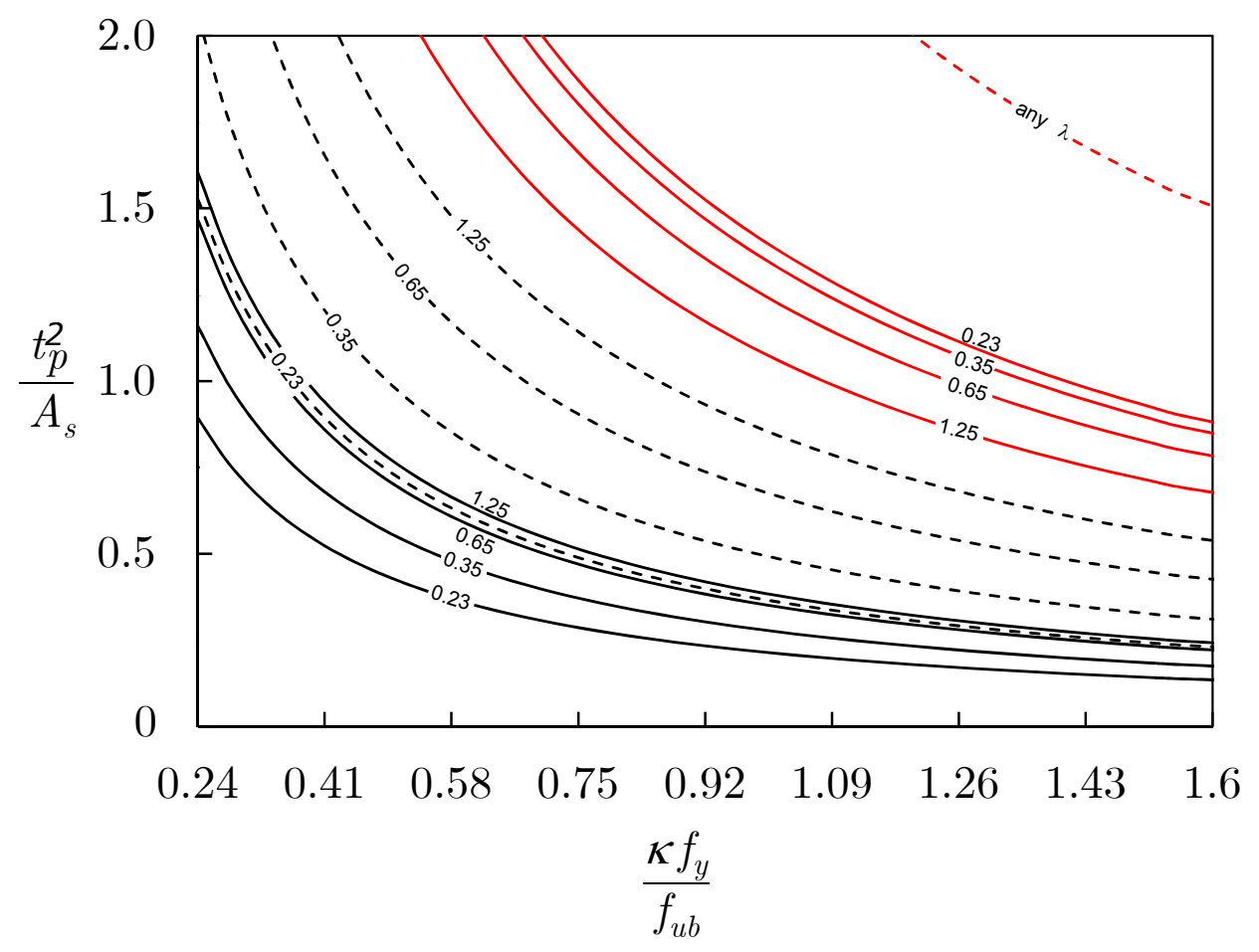

FIG. 5. Ultimate failure map. Black and red isolines are régime boundaries at mode $1 \rightarrow 2$ and mode $2 \rightarrow 3$ transition, respectively. Solid lines for non-circular and dotted lines for beam yield line patterns. 


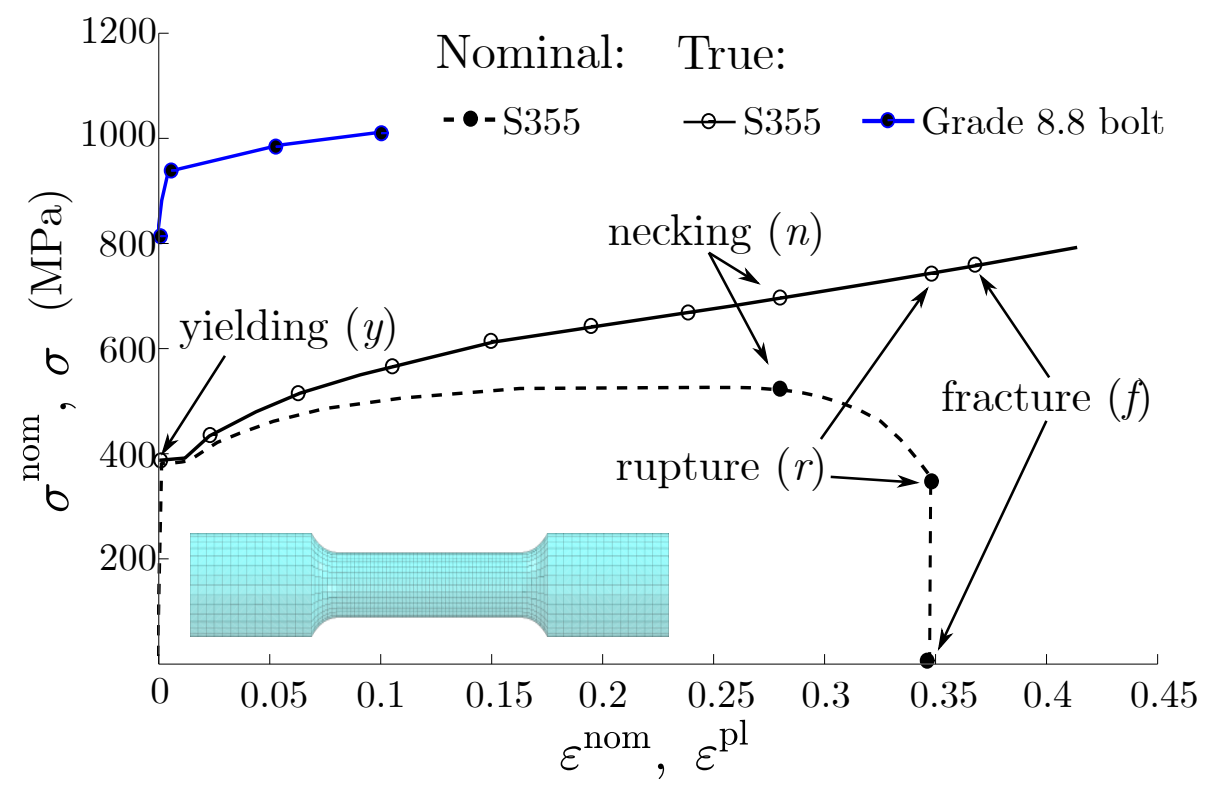

FIG. 6. Material properties for S355 steel and Grade 8.8 bolt. 


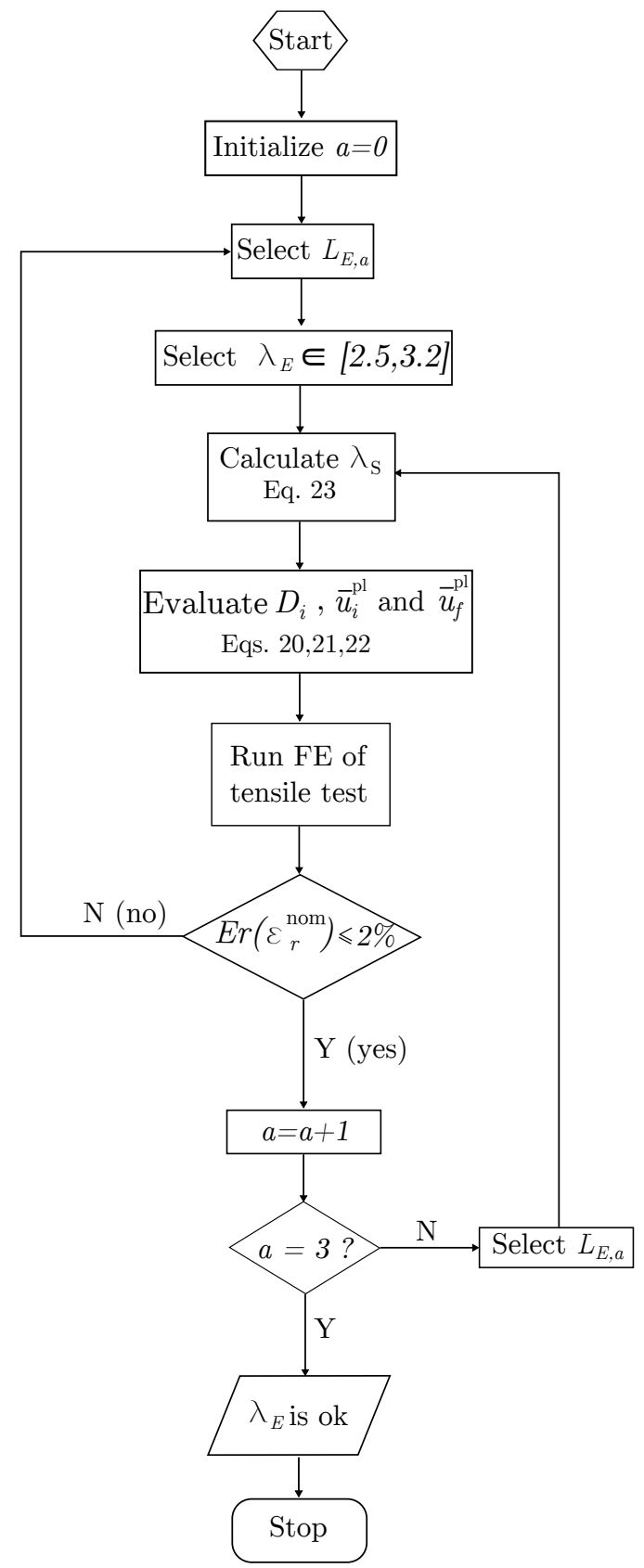

FIG. 7. Flow-chart on the procedure to calibrate $\lambda_{E}$. 


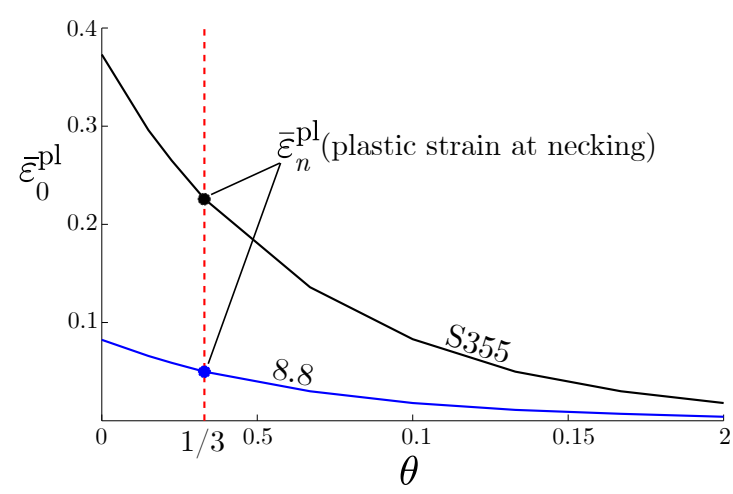

(a)

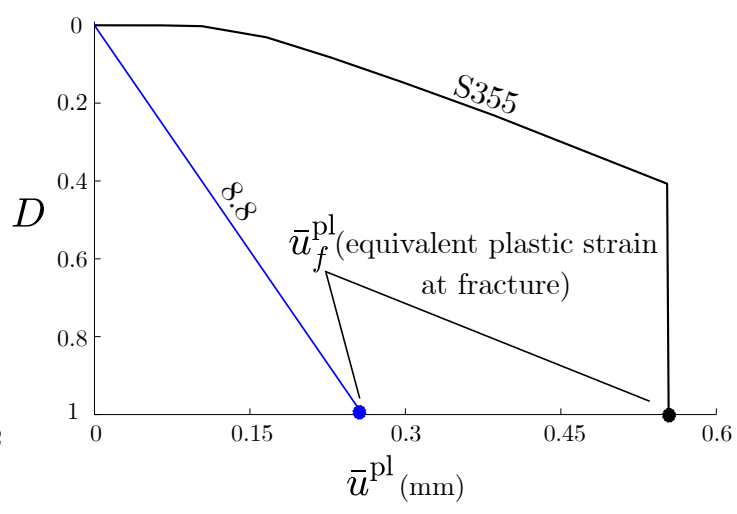

(b)

FIG. 8. (a) Damage initiation criterion and (b) damage evolution law. 


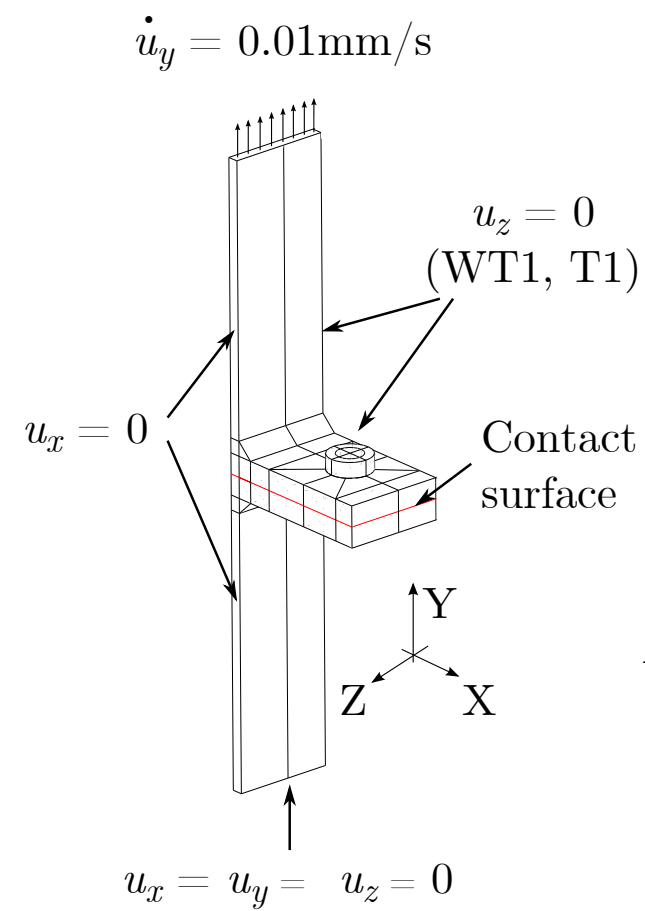

(a)

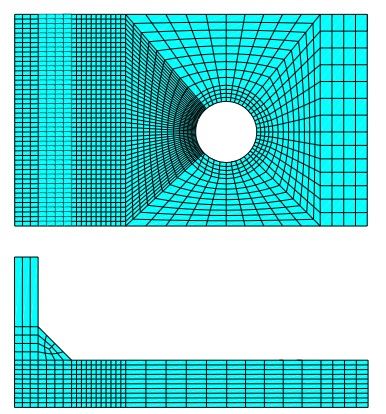

(c)

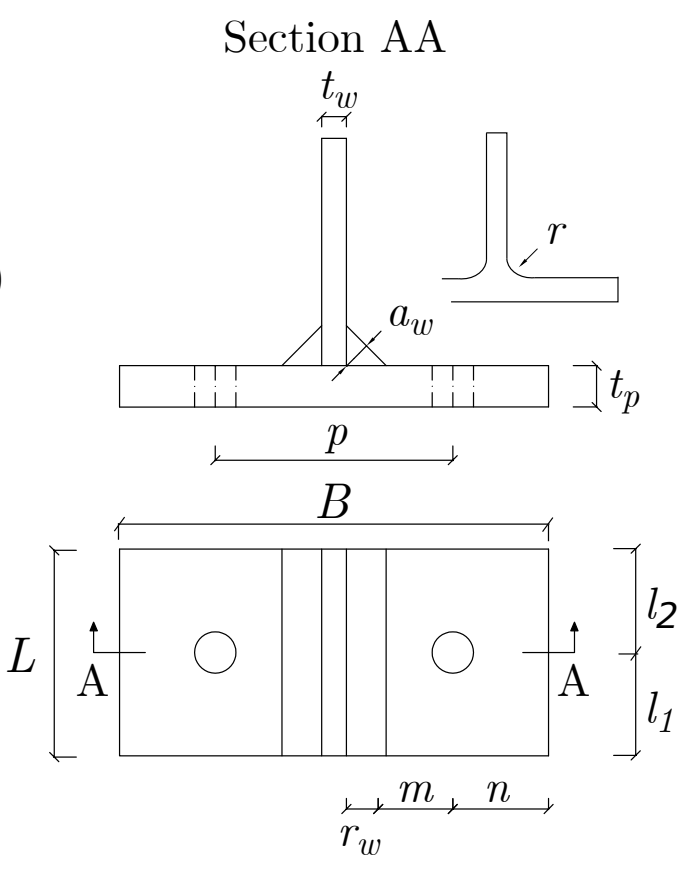

(b)

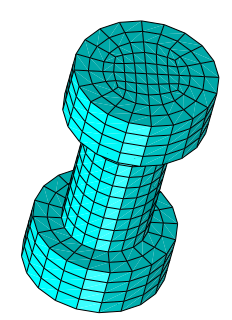

Shaft section

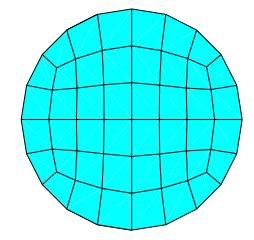

(d)

FIG. 9. (a) FE model of the T-stubs and (b) its corresponding geometric parameters. The FE meshes for bolt and flange are shown in (c) and (d). $r_{w}=0.8 a_{w} \sqrt{2}$ for WT1 and T15; $r_{w}=0.8 r$ for T1. 


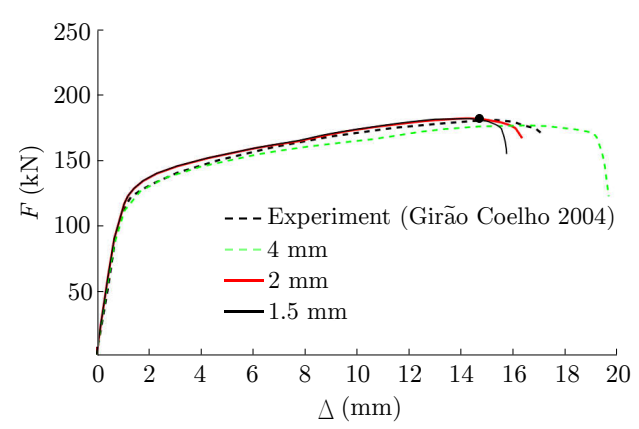

(a)

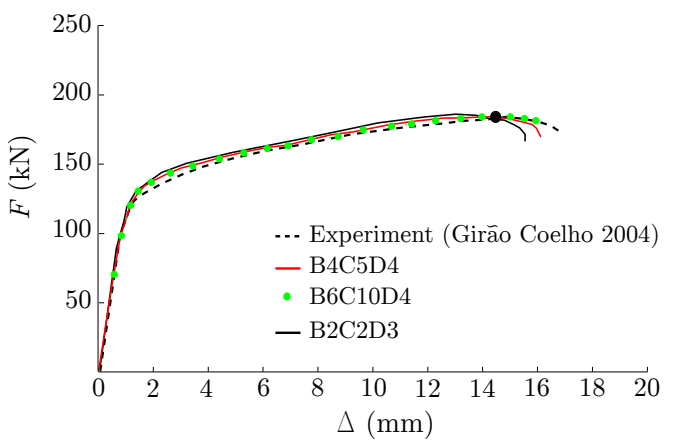

(c)

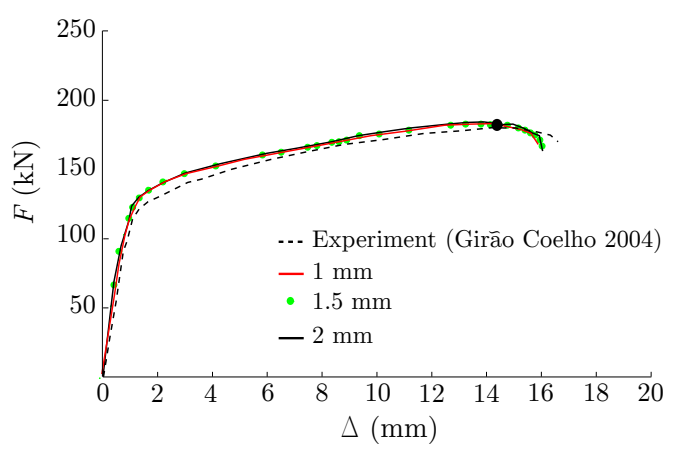

(b)

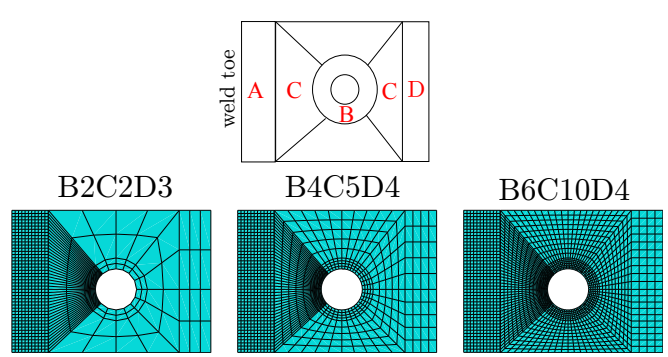

(d)

FIG. 10. Results of mesh sensitivity study for WT1 (Girão Coelho 2004), where black dot indicates ultimate displacement $\Delta_{u}$ : (a) effects of element size in the bolt shaft; (b) effects of element size at the weld toe; (c) predicted $\mathrm{F}-\Delta$ curves by modelling the flange as shown in (d). 


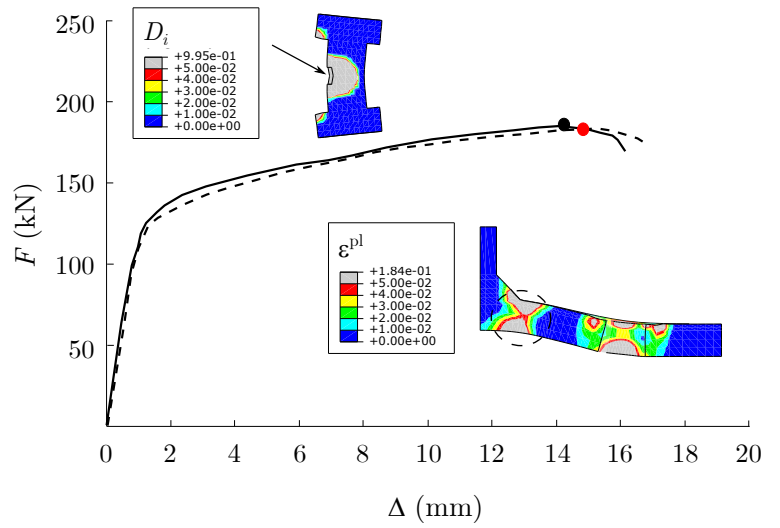

(a) WT1 (Girao Coelho 2004)

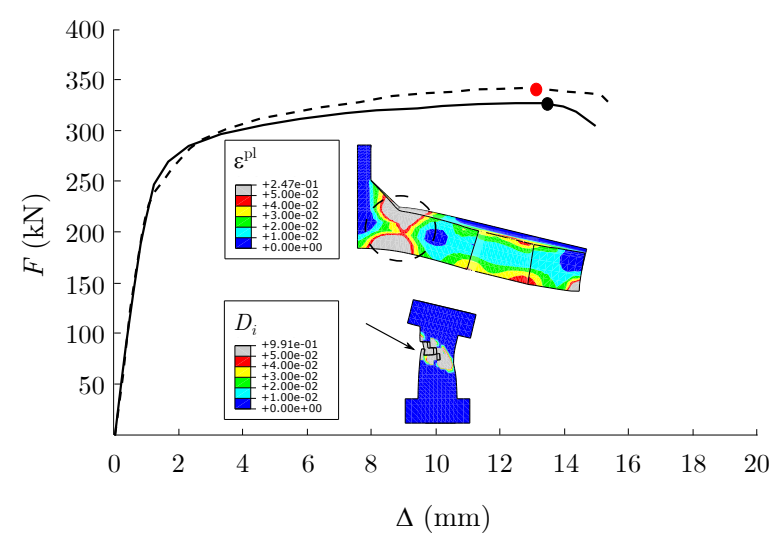

(c) T15 (Ribeiro et al. 2015)

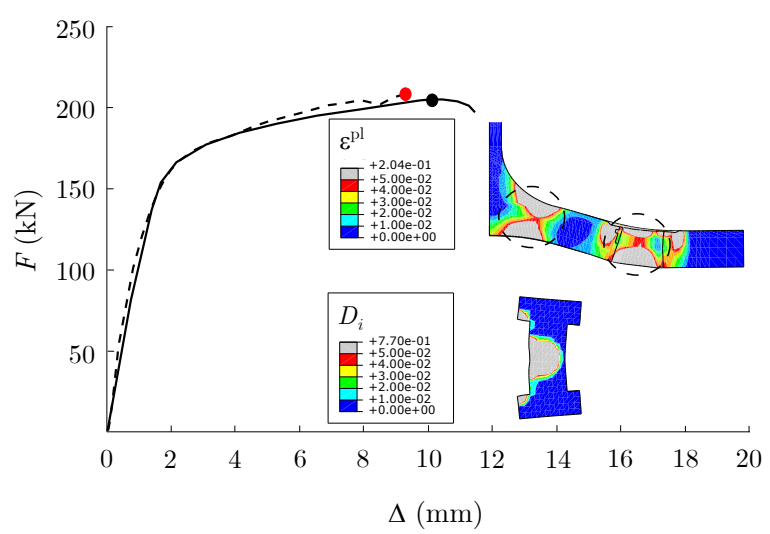

(b) T1 (Bursi and Jaspart 1997)

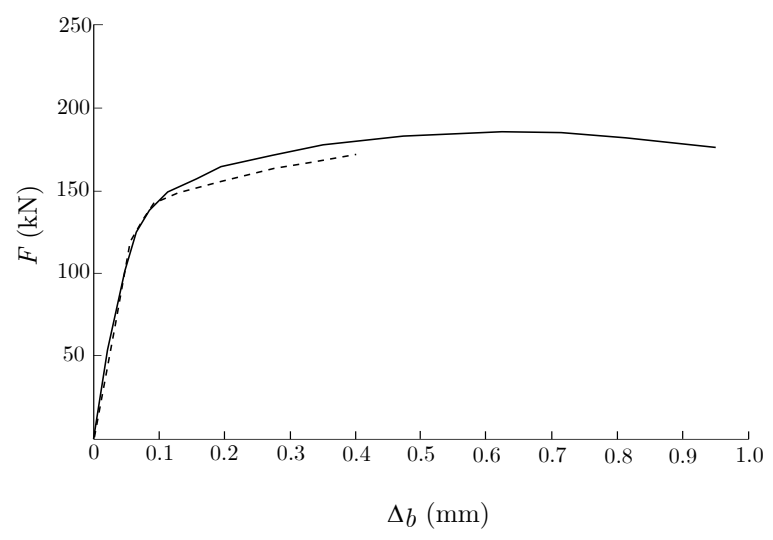

(d) Force - bolt elongation (Girao Coelho 2004)

FIG. 11. Comparison between FE (-) and experiments (- - ). Arrow indicates deleted elements in the bolt and dotted circle indicates where plastic hinge develops. 


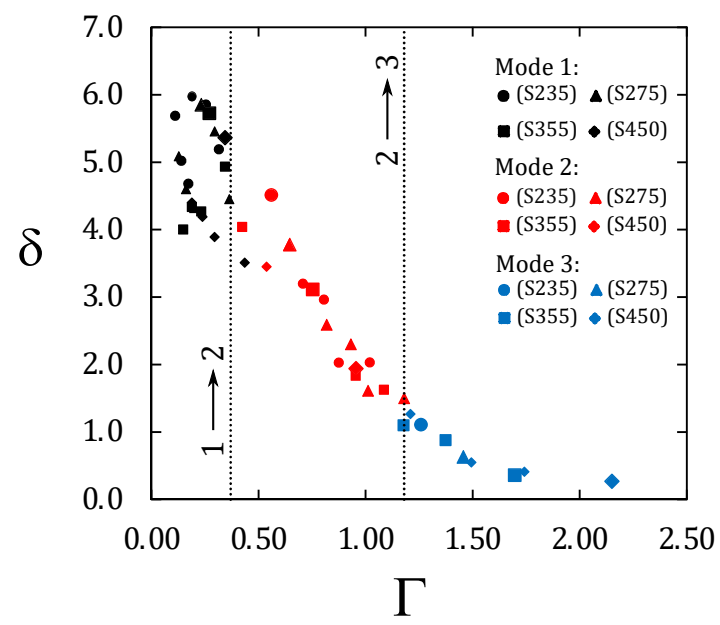

(a)

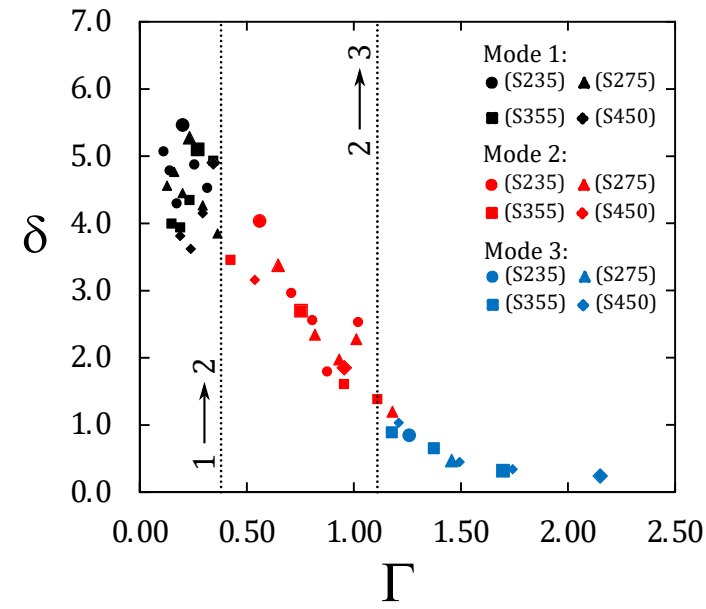

(b)

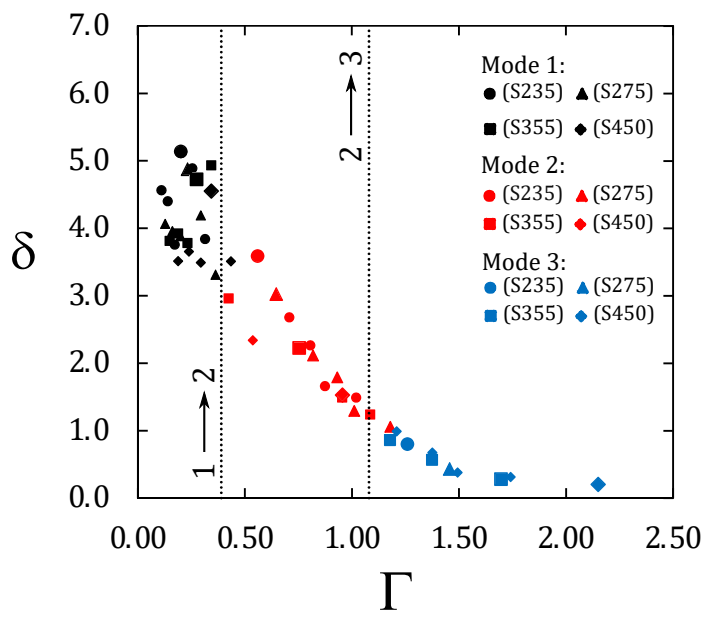

(c)

FIG. 12. Results of parametric study: (a) $\lambda_{\min }=0.9$, (b) $\lambda_{\text {inter }}=1.11$ and (c) $\lambda_{\max }=1.25$. Vertical lines indicate boundaries between ultimate failure modes. 

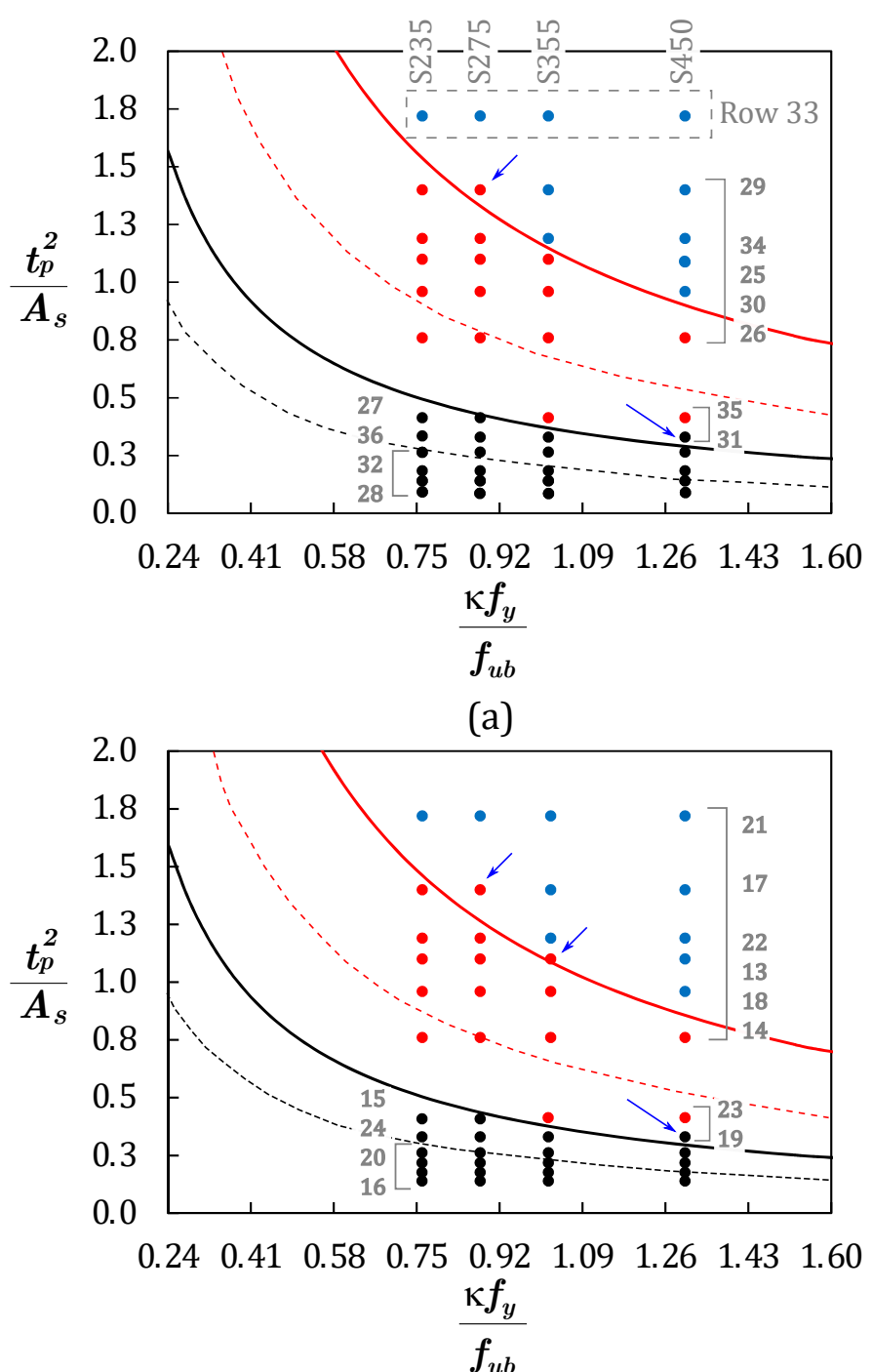

(b)

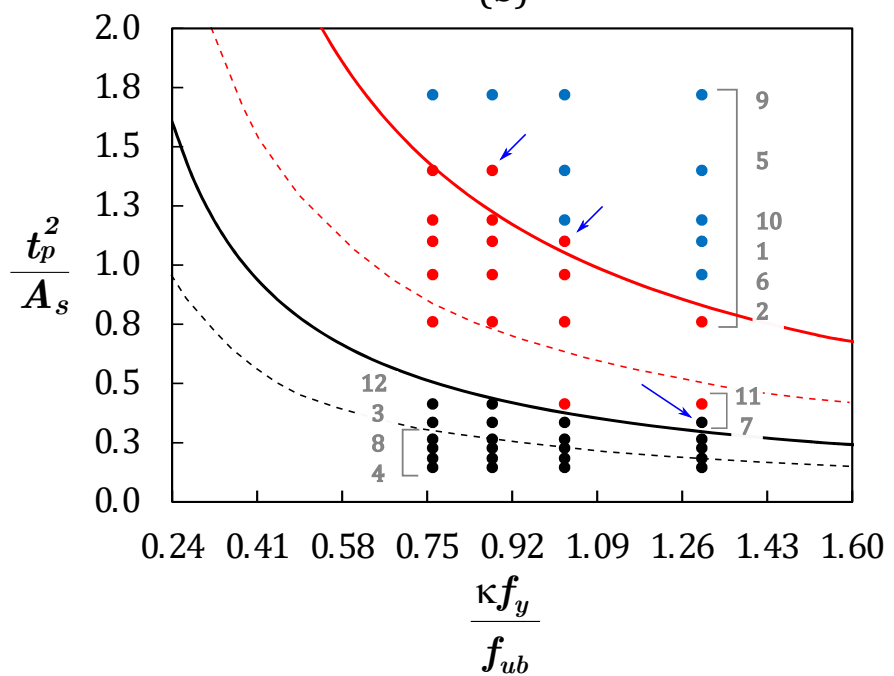

(c)

FIG. 13. Predicted failure mode by FE for (a) $\lambda_{\min }=0.9$, (b) $\lambda_{\text {inter }}=1.11$ and (c) $\lambda_{\max }=1.25$. Mode $1(\bullet)$, Mode $2(\bullet)$ and Mode $3(\bullet)$. Grey numbers identify the geometry (Table 4) and flange materials are indicated. 


\begin{tabular}{cc}
\hline Mode transition & $\beta$ \\
\hline $1 \rightarrow 2$ & $\frac{2 \lambda}{1+2 \lambda}$ \\
$2 \rightarrow 3$ & 2 \\
\hline
\end{tabular}

TABLE 1: Values of $\beta$ delineating the transition between failure modes given by Piluso et al. (2001). 


\begin{tabular}{lcccc}
\hline & & \multicolumn{2}{c}{$h(\lambda)$} & \\
\hline & Mode transition & Non-circular & Beam & $\kappa$ \\
\hline \multirow{2}{*}{ Plastic limit state } & $1 \rightarrow 2$ & $\frac{3.57 \lambda}{(1+2 \lambda)(4+1.25 \lambda)}$ & $\frac{1.44 \lambda}{(1+2 \lambda)}$ & 1 \\
& $2 \rightarrow 3$ & $\frac{3.57}{4+1.25 \lambda}$ & 1.44 & 1 \\
\hline Ultimate limit state & $1 \rightarrow 2$ & $\frac{6 \lambda}{\left(4+\frac{1.25 \lambda)(1+2 \lambda)}{6}\right.}$ & $\frac{2.4 \lambda}{1+2 \lambda}$ & Eq. 10 \\
& $2 \rightarrow 3$ & $\frac{(4+1.25 \lambda)}{2}$ & 2.4 & Eq. 10 \\
\hline
\end{tabular}

TABLE 2: Expressions for $h(\lambda)$ in Eq. 13 . 


\begin{tabular}{lcccccccccccc}
\hline Model & $m$ & $n$ & $t_{p}$ & $d_{b}$ & $l_{1}$ & $l_{2}$ & $L$ & $B$ & $p$ & $a_{w} / r$ & $t_{w}$ & $r_{w}$ \\
\hline WT1 & 34.34 & 30 & 10 & 12 & 20 & 25 & 45 & 150.08 & 50 & 5 & 10 & 5.7 \\
T1 & 29.45 & 30 & 10.7 & 12 & 20 & 20 & 40 & 150 & 90 & 15 & 7.1 & 12 \\
T15 & 42.1 & 30 & 15 & 20 & 52.5 & 52.5 & 105 & 170 & 110 & 7 & 10 & 7.9 \\
\hline
\end{tabular}

TABLE 3: Geometric dimensions corresponding to Fig. 9b. All dimensions are in mm except $a_{W} / r$. 


\begin{tabular}{|c|c|c|c|c|c|c|c|c|c|}
\hline \multirow[b]{2}{*}{ Row } & \multicolumn{5}{|c|}{ Geometry } & \multicolumn{4}{|c|}{ Predicted $\delta$} \\
\hline & $\mathrm{m}$ & $\mathrm{n}$ & $\lambda$ & $t_{p}$ & $d_{b}$ & $\mathrm{~S} 235$ & $\mathrm{~S} 275$ & S355 & $\mathrm{S} 450$ \\
\hline 1 & 40 & 50 & 1.25 & 8 & 10 & $2.3(5.0,2.0)$ & $1.8(3.8,1.6)$ & $1.2(1.4,0.7)$ & $0.7(0.9,0.5)$ \\
\hline 2 & 40 & 50 & 1.25 & 8 & 12 & $3.6(5.0,2.3)$ & $3.0(3.8,2.1)$ & $2.2(3.6,2.6)$ & $1.5(2.6,1.57)$ \\
\hline 3 & 40 & 50 & 1.25 & 8 & 20 & $5.1(5.2,5.1)$ & $4.9(4.0,3.8)$ & $4.7(3.9,3.7)$ & $4.6(3.34,3.53)$ \\
\hline 4 & 40 & 50 & 1.25 & 8 & 27 & $4.6(5.2,5.0)$ & $4.1(4.0,3.6)$ & $3.8(3.9,3.5)$ & $3.5(3.34,2.65)$ \\
\hline 5 & 40 & 50 & 1.25 & 9 & 10 & $1.5(1.9,1.4)$ & $1.1(1.2,0.4)$ & $0.6(0.3,0.3)$ & $0.3(0.4,0.18)$ \\
\hline 6 & 40 & 50 & 1.25 & 9 & 12 & $2.7(4.0,1.4)$ & $2.1(2.5,1.4)$ & $1.5(1.5,1.4)$ & $1.0(0.92,0.35)$ \\
\hline 7 & 40 & 50 & 1.25 & 9 & 20 & $4.9(4.1,4.1)$ & $4.2(3.2,4.6)$ & $4.9(3.1,3.7)$ & $3.5(2.6,2.1)$ \\
\hline 8 & 40 & 50 & 1.25 & 9 & 27 & $4.4(4.1,3.9)$ & $4.0(3.2,2.3)$ & $3.9(3.1,2.7)$ & $3.7(2.7,2.16)$ \\
\hline 9 & 40 & 50 & 1.25 & 10 & 10 & $0.8(0.3,0.2)$ & $0.4(1.1,0.2)$ & $0.3(0.5,0.3)$ & $0.2(0.3,0.3)$ \\
\hline 10 & 40 & 50 & 1.25 & 10 & 12 & $1.7(3.2,1.4)$ & $1.3(1.3,1.1)$ & $0.9(0.7,0.3)$ & $0.4(0.1,0.2)$ \\
\hline 11 & 40 & 50 & 1.25 & 10 & 20 & $3.8(3.4,3.9)$ & $3.3(2.6,4.2)$ & $3.0(3.1,2.5)$ & $2.3(2.5,3.0)$ \\
\hline 12 & 40 & 50 & 1.25 & 10 & 27 & $4.1(3.4,3.2)$ & $3.9(2.6,2.4)$ & $3.8(2.6,2.3)$ & $3.5(2.2,3.5)$ \\
\hline 13 & 44 & 46 & 1.1 & 8 & 10 & 2.6 & 2.01 & 1.4 & 0.9 \\
\hline 14 & 44 & 46 & 1.1 & 8 & 12 & 4.0 & 3.4 & 2.7 & 2.1 \\
\hline 15 & 44 & 46 & 1.1 & 8 & 20 & 5.5 & 5.3 & 5.1 & 4.9 \\
\hline 16 & 44 & 46 & 1.1 & 8 & 27 & 5.1 & 4.6 & 4.0 & 3.8 \\
\hline 17 & 44 & 46 & 1.1 & 9 & 10 & 2.5 & 2.3 & 0.5 & 0.3 \\
\hline 18 & 44 & 46 & 1.1 & 9 & 12 & 3.0 & 1.6 & 1.3 & 0.3 \\
\hline 19 & 44 & 46 & 1.1 & 9 & 20 & 5.6 & 5.4 & 5.3 & 5.0 \\
\hline 20 & 44 & 46 & 1.1 & 9 & 27 & 4.8 & 4.8 & 3.9 & 3.6 \\
\hline 21 & 44 & 46 & 1.1 & 10 & 10 & 1.2 & 0.9 & 0.5 & 0.3 \\
\hline 22 & 44 & 46 & 1.1 & 10 & 12 & 1.8 & 2.3 & 0.8 & 0.7 \\
\hline 23 & 44 & 46 & 1.1 & 10 & 20 & 4.5 & 3.9 & 1.9 & 3.5 \\
\hline 24 & 44 & 46 & 1.1 & 10 & 27 & 4.7 & 4.5 & 4.4 & 4.2 \\
\hline 25 & 47 & 43 & 0.9 & 8 & 10 & 3.0 & 2.3 & 1.6 & 1.1 \\
\hline 26 & 47 & 43 & 0.9 & 8 & 12 & 4.5 & 3.8 & 3.1 & 1.9 \\
\hline 27 & 47 & 43 & 0.9 & 8 & 20 & 6.0 & 5.9 & 5.7 & 5.4 \\
\hline 28 & 47 & 43 & 0.9 & 8 & 27 & 5.7 & 5.1 & 4.0 & 4.4 \\
\hline 29 & 47 & 43 & 0.9 & 9 & 10 & 2.0 & 2.6 & 0.3 & 0.6 \\
\hline 30 & 47 & 43 & 0.9 & 9 & 12 & 3.2 & 1.8 & 1.3 & 0.4 \\
\hline 31 & 47 & 43 & 0.9 & 9 & 20 & 5.9 & 5.5 & 4.9 & 3.5 \\
\hline 32 & 47 & 43 & 0.9 & 9 & 27 & 5.0 & 4.6 & 4.3 & 4.2 \\
\hline 33 & 47 & 43 & 0.9 & 10 & 10 & 1.5 & 0.6 & 0.4 & 1.1 \\
\hline 34 & 47 & 43 & 0.9 & 10 & 12 & 2.0 & 1.6 & 1.0 & 0.9 \\
\hline 35 & 47 & 43 & 0.9 & 10 & 20 & 5.2 & 4.5 & 4.0 & 3.5 \\
\hline 36 & 47 & 43 & 0.9 & 10 & 27 & 4.7 & 4.3 & 4.3 & 3.9 \\
\hline
\end{tabular}

TABLE 4: Dimensions (mm) of T-stubs - see Fig. $9 \mathrm{~b}$ - considered in the parametric study. $l_{1}=l_{2}=L / 2=125 \mathrm{~mm} ; B=200 \mathrm{~mm} ; t_{w}=6.5 \mathrm{~mm} ; a_{w}=5 \mathrm{~mm}$ and $r_{w}=7.5 \mathrm{~mm}$ in all the models. XX (YY, ZZ) refers to FEM (Piluso et al. (2001), Francavilla et al. (2016)). 


\begin{tabular}{ccccccccccc}
\hline Steel & $\begin{array}{c}\sigma_{y} \\
(\mathrm{MPa})\end{array}$ & $\begin{array}{c}\sigma_{u} \\
(\mathrm{MPa})\end{array}$ & $\varepsilon_{y}$ & $\varepsilon_{h}$ & $\varepsilon_{u}$ & $\varepsilon_{f}$ & $\begin{array}{c}E \\
(\mathrm{MPa})\end{array}$ & $\begin{array}{c}E_{h} \\
(\mathrm{MPa})\end{array}$ & $\begin{array}{c}E_{f} \\
(\mathrm{MPa})\end{array}$ & $\kappa$ \\
\hline S235 & 235 & 360 & 0.001 & 0.014 & 0.036 & 0.25 & 210000 & 5500 & 360 & 2.587 \\
$\mathrm{~S} 275$ & 275 & 430 & 0.001 & 0.015 & 0.047 & 0.22 & 210000 & 4800 & 430 & 2.560 \\
$\mathrm{~S} 355$ & 355 & 510 & 0.002 & 0.017 & 0.053 & 0.2 & 210000 & 4250 & 510 & 2.305 \\
$\mathrm{~S} 450$ & 440 & 550 & 0.002 & 0.017 & 0.053 & 0.17 & 210000 & 4250 & 550 & 2.305 \\
\hline
\end{tabular}

TABLE 5: Key stresses, strains, moduli and $\kappa$ corresponding to Fig. 3. 


\begin{tabular}{cc|cc|cc|cc}
\hline \multicolumn{2}{c}{$\mathrm{S} 235$} & \multicolumn{2}{c}{$\mathrm{S} 275$} & \multicolumn{2}{c}{$\mathrm{S} 355$} & \multicolumn{2}{c}{$\mathrm{S} 450$} \\
\hline$\theta$ & $\bar{\varepsilon}_{0}^{\mathrm{pl}}$ & $\theta$ & $\bar{\varepsilon}_{0}^{\mathrm{pl}}$ & $\theta$ & $\bar{\varepsilon}_{0}^{\mathrm{pl}}$ & $\theta$ & $\bar{\varepsilon}_{0}^{\mathrm{pl}}$ \\
\hline 0.1 & 0.183 & 0.1 & 0.158 & 0.1 & 0.144 & 0.1 & 0.128 \\
0.13 & 0.176 & 0.13 & 0.156 & 0.13 & 0.143 & 0.13 & 0.130 \\
0.33 & 0.130 & 0.33 & 0.112 & 0.33 & 0.102 & 0.33 & 0.090 \\
0.67 & 0.078 & 0.67 & 0.067 & 0.67 & 0.061 & 0.67 & 0.054 \\
1 & 0.048 & 1 & 0.041 & 1 & 0.037 & 1 & 0.033 \\
1.33 & 0.029 & 1.33 & 0.025 & 1.33 & 0.022 & 1.33 & 0.020 \\
1.67 & 0.114 & 1.67 & 0.015 & 1.67 & 0.014 & 1.67 & 0.012 \\
2 & 0.010 & 2 & 0.009 & 2 & 0.008 & 2 & 0.007 \\
\hline
\end{tabular}

TABLE 6: Damage initiation criteria. 


\begin{tabular}{ccccccccccc}
\hline & \multicolumn{3}{c}{ Geometry } & \multicolumn{3}{c}{ Material } & \multicolumn{3}{c}{ Failure Mode } \\
\hline Model & $t_{p}$ & $d_{b}$ & $\lambda$ & $\kappa$ & $f_{y}$ & $f_{u b}$ & Exp. & Plas. & Ult. \\
\hline 1 & 14.4 & 20 & 1.04 & 3.12 & 291 & 800 & 2 & 2 & 2 \\
2 & 14.6 & 20 & 1.04 & 3.33 & 265 & 800 & 2 & 2 & 2 \\
3 & 13 & 20 & 0.52 & 3.49 & 273 & 800 & 2 & 2 & 2 \\
4 & 12.3 & 24 & 1.19 & 3.68 & 300 & 800 & 1 & 2 & 1 \\
6 & 16.4 & 24 & 0.99 & 3.70 & 280 & 800 & 2 & 2 & 2 \\
9 & 12.5 & 27 & 0.99 & 3.58 & 301 & 800 & 1 & 1 & 1 \\
12 & 12.2 & 20 & 0.93 & 2.95 & 347 & 800 & 1 & 2 & 1 \\
\hline WT1 & 10 & 12 & 0.9 & 2.31 & 355 & 800 & 1 & 2 & 2 \\
WT7_M20 & 10 & 20 & 0.9 & 3.33 & 355 & 800 & 1 & 2 & 1 \\
WT7_M16 & 10 & 16 & 0.9 & 3.33 & 355 & 800 & 1 & 2 & 2 \\
WT57_M16 & 10 & 16 & 0.9 & 1.74 & 690 & 800 & 2 & 2 & 2 \\
WT57_M12 & 10 & 12 & 0.9 & 1.74 & 690 & 800 & 2 & 2 & 2 \\
WT7_M12 & 10 & 12 & 0.9 & 3.33 & 355 & 800 & 2 & 2 & 2 \\
\hline T1 & \multicolumn{1}{c}{1.02} & & & & 1 & 2 & 2 \\
\hline T15 & & 0.7 & & & & 2 & 2 \\
\hline
\end{tabular}

TABLE 7: Comparison of predicted failure mode (by the deformation maps) against experiment data from (a) Piluso et al. (2001), (b) Girão Coelho (2004), (c) Bursi and Jaspart (1997) and (d) Ribeiro et al. (2015). 